\title{
10 Fuzzy reliability of system using different types of bifuzzy failure rates of components
}

\begin{abstract}
The present study proposes to determine the fuzzy reliability of systems using different types of conflicting bifuzzy numbers Till now, to evaluate the fuzzy reliability used the failure rates of components of systems follow the same type of fuzzy set or conflicting bifuzzy set. However, in real-life problems, such type of situation rarely occurs. Therefore, in this study, a new method has been introduced to determine the fuzzy reliability of a system having components following different types of conflicting bifuzzy failure rates. Using the introduced method, membership functions and nonmembership functions of fuzzy reliability of a series, parallel, parallel-series, and series-parallel systems are evaluated. Numerical problems are also taken to describe the proposed study.
\end{abstract}

Keywords: fuzzy system reliability, conflicting bifuzzy number, failure rate, system

\subsection{Introduction}

The traditional reliability of an item is the probability that item will perform a required function without failure under stated conditions for a stated period of time. More generally, reliability is the capacity of parts, components, equipments, products, and systems to perform their required functions for desired periods of time without failure, in specified environments and with a desired confidence. However, in real-life problems, the collected data or information are not always accurate hence the deduction of appropriate value of probability becomes very difficult in many cases. To handle this problem, fuzzy set theory has been used to analyze the reliability of a system. The fuzzy set theory introduced by Zadeh [1].

To understand the concept of fuzzy set, let us consider a system having three independent components. Evidently, the system is in working stage when all three components are in working condition simultaneously. However, when one or two components are failed, the system will operate in an offended state. In this stage, the system is neither fully working nor fully failed, but is in some intermediate state. This implies that the binary state assumption for delineate system failure and

Pawan Kumar, Department of Mathematics, Statistics and Computer Science

S.B. Singh, G.B. Pant University of Agriculture and Technology, Pantnagar, Uttarakhand, India

Ә Open Access. (C) 2020 Pawan Kumar, S. B. Singh, published by De Gruyter. (c) BY-NC-ND This work is licensed under a Creative Commons Attribution-NonCommercial-NoDerivatives 4.0 International License.

https://doi.org/10.1515/9783110628616-010 
success may no longer be suitable. To handle this problem, we fuzzify the system failure. In real-life problems a person may assume that a commodity belongs to a set to a few degrees, but he is not sure that a commodity belongs to a set. In other words, there may be a hesitance or uncertainty about the membership function of object in $A$. An eventually solution is to use intuitionistic fuzzy sets (IFS), defined by Atanassov (1986).

The concept of IFS is the generalizations of the fuzzy set theory of Zadeh [1]. The membership function is considered only in fuzzy set but membership degree $\mu_{A}(x)$ and nonmembership function $\nu_{A}(x)$ are considered in IFS and the sum of membership and nonmembership degree is less than or equal to 1 . Suppose if the sum of the value of membership and nonmembership function is greater than 1 then how do we fulfill the condition, for example, in a case where the membership function $\mu_{A}(x)=0.8$ and the nonmembership function $v_{A}(x)$ equals to 0.6 ? This situation gives us a chance to redefine a solution lastly to reform the concept of IFS. Based on this reason Zamali et al. [2] gave a new concept of conflicting bifuzzy set (CBFS). In bifuzzy set, the sum of membership and nonmembership degree is less than 2. Hence, CBFS theory is an extension of IFS theory. Imran et al. [3] presented the definition and graphical representation of conflicting bifuzzy sets based on intuitionistic evaluation.

Fuzzy reliability theory was introduced and developed by Wen et al. [4, 5], Chen and Mon [6], Onisawa and Kacprzyk [7], and Cai [8]. The recent compilation of papers by Onisawa and Kacprzyk [7] gave several different approaches for fuzzy reliability. Kumar and Yadav [9] proposed a new method for analyzing fuzzy reliability of systems using different types of intuitionistic fuzzy failure rates of components. Aliev et al. [10] analyzed fuzzy reliability of systems using time dependent fuzzy set of components. Mon and Cheng [11] developed a new method for fuzzy reliability of systems for components with different membership functions. Kumar et al. [12] developed a new method for evaluating fuzzy reliability using intuitionistic fuzzy number. Mon et al. [11] analyzed the fuzzy reliability of different systems with different membership functions.

In the above-mentioned studies, it is supposed that the failure rates of components of systems follow the conflicting bifuzzy numbers (CBFNs) to evaluate the fuzzy reliability of systems. In this study, we have constructed the membership degree and nonmembership degree of the fuzzy system reliability using different types of CBFNs. Using introduced method, membership degree and non-membership degree of fuzzy system reliability of series, parallel, parallel-series, and series-parallel systems are evaluated. Numerical problems are also taken to exemplify the proposed study. 


\subsection{Preliminaries}

Definition 10.1 ([1]). If a set $Y$ be fixed, then a fuzzy set $\tilde{Z}$ is given by

$$
\bar{Z}=\left\{\left\langle y, \mu_{\tilde{Z}}(y)\right\rangle: y \in Y\right\}
$$

where $\mu_{\tilde{Z}}(x) \in[0,1]$ is the membership function of the element $y \in \mathrm{Y}$.

Definition 10.2 (Atanassov 1986). If a set $Z$ be fixed then an intuitionistic fuzzy set (IFS) $\tilde{A}$ is given by

$$
\tilde{A}=\left\{\left\langle z, \mu_{\tilde{A}}(z), v_{\tilde{A}}(z): z \in Z\right\rangle\right\}
$$

where $\mu_{\tilde{A}}: Z \rightarrow[0,1]$ is the membership function of positive $z,(z \in Z)$ and $v_{\tilde{A}}: Z \rightarrow[0,1]$ is the non- membership function of negative $z,(z \in Z)$ w.r.t. $\tilde{A}$ and $0 \leq \mu_{\tilde{A}}+v_{\tilde{A}} \leq 1$.

Zamali [2] in his study proposed the new notion called a bifuzzy set which is extension of IFS. He defines CBFS.

Definition 10.3 ([2]). If a set $A$ is fixed then a CBFS $\tilde{Z}$ of $A$ is an object having the given form as follows

$$
\tilde{Z}=\left\{\left\langle a, \mu_{\tilde{Z}}(a), v_{\tilde{Z}}(a): a \in A\right\rangle\right\}
$$

where $\mu_{\tilde{Z}}: A \rightarrow[0,1]$ is the membership function of positive $a,(a \in A)$ with respect to $\tilde{Z}$ and $v_{\tilde{Z}}: A \rightarrow[0,1]$ is the nonmembership function of negative $a,(a \in A)$ with respect to $\tilde{Z}$ and $0 \leq \mu_{\tilde{Z}}(a)+v_{\tilde{Z}}(a) \leq 2$.

\subsection{1 $\alpha$-Level set or $\alpha$-Cut set of a CBFS}

$\alpha$-Cut of a CBFS $\tilde{A}$ is defined as

$$
\tilde{A}_{\alpha}=\left\{x \in X: \mu_{\tilde{A}}(x) \geq \alpha,\right\} ; 0 \leq \alpha
$$

\subsection{2 $\beta$-Level set or $\beta$-Cut set of a CBFS}

$\beta$-Cut of a CBFS $\tilde{A}$ is defined as

$$
\tilde{A}_{\beta}=\left\{x \in X: v_{\tilde{A}}(x) \leq \beta\right\} ; \beta \leq 1
$$




\subsubsection{Triangular CBFS}

A triangular CBFS $\tilde{A}$ is defined as

$$
\tilde{A}=\left(m-l, m, m+n ; m-l^{\prime}, m, m+n^{\prime}\right)
$$

where $m \in R$ is the center, $l>0$ and $n>0$ are the left and right dispersion of the membership function of $\tilde{A}$, respectively. $l^{\prime}>0$ and $n^{\prime}>0$ are the left and right spreads of the nonmembership function of $\tilde{A}$, respectively.

\subsubsection{Trapezoidal CBFS}

$$
\tilde{A}=\left(p-l, q, r, s+n ; p-l^{\prime}, q, r, s+n^{\prime}\right)
$$

where $p, q, r, s \in R$ is the center, $l>0$ and $n>0$ are the left and right dispersion of the $\mu_{\tilde{A}}(x)$, respectively. $l^{\prime}>0$ and $n^{\prime}>0$ are the left and right dispersion of the $v_{\tilde{A}}(x)$, respectively.

\subsection{Bifuzzy reliability function or survival function}

The reliability function $R(t)$ of systems in terms of failure rate $\lambda(t)$ is given by following formula

$$
R(t)=\exp \left[-\int_{0}^{t} \lambda\left(t^{\prime}\right) d t^{\prime}\right], \quad t>0
$$

Let us consider the failure rate is constant, that is, $(t)=\lambda$, then the reliability is as follows:

$$
R(t)=\exp (-\lambda t)
$$

If failure rate is not precise, then failure rate is considered as CBFS.

Let failure rate is considered as $\operatorname{CBFS} \tilde{\lambda}$ defined on $Z$ given below:

$$
\tilde{\lambda}=\left\{<z, \mu_{\tilde{\lambda}}(z), v_{\tilde{\lambda}}(z)>: z \in Z\right\}
$$

Two types of cuts for CBFS are defined as follows:

$\alpha$-Cut of membership function $\mu_{\tilde{\lambda}}(z)$ which is defined as

$$
\tilde{\lambda}_{\alpha}=\left\{z: \mu_{\tilde{\lambda}}(z) \geq \alpha, \alpha \in[0,1]\right\}
$$


$\beta$-Cut of nonmembership function $v_{\tilde{\lambda}}(z)$ is as

$$
\tilde{\lambda}_{\beta}=\left\{z: v_{\tilde{\lambda}}(z) \leq \beta, \beta \in[0,1]\right\}
$$

Failure rate $\tilde{\lambda}$ is in form of CBFS. Then fuzzy-convexity of CBFS is as follows:

$$
\tilde{\lambda}_{\alpha}=\left[\lambda_{\alpha}^{L}, \lambda_{\alpha}^{R}\right] \quad \forall \alpha \in[0,1]
$$

Also fuzzy-concavity of CBFS is as follows:

$$
\tilde{\lambda}_{\beta}=\left[\lambda_{\beta}^{L}, \lambda_{\beta}^{R}\right] \quad \forall \beta \in[0,1]
$$

where $\lambda_{\alpha}^{L}$ and $\lambda_{\alpha}^{R}$ increasing and decreasing function of $\alpha$ and $\beta$, respectively. $\lambda_{\beta}^{L}$ and $\lambda_{\beta}^{R}$ increasing and decreasing function of $\alpha$ and $\beta$, respectively, with $\alpha, \beta \in[0,1]$.

The reliability function $\bar{R}(t)$ using CBFS defined on $X$ is as follows:

$$
\bar{R}(t)=\left\{<x, \mu_{\bar{R}(t)}(x), v_{\bar{R}(t)}(x)>: x \in X\right\}
$$

Now failure rate follows CBFS, then the reliability $\bar{R}(t)$ is also in the form of CBFS.

Now $\alpha$-Cut for membership function of $\bar{R}(t)$ is

$$
\bar{R}_{\alpha}(t)=\left[r_{\alpha}^{L}(t), r_{\alpha}^{R}(t)\right]
$$

where

$$
\begin{array}{ll}
r_{\alpha}^{L}(t)=\min \left(e^{-x t}\right) & \text { s.t. } \lambda_{\alpha}^{L} \leq x \leq \lambda_{\alpha}^{R} \\
r_{\alpha}^{R}(t)=\max \left(e^{-x t}\right) & \text { s.t. } \lambda_{\alpha}^{L} \leq x \leq \lambda_{\alpha}^{R}
\end{array}
$$

$\beta$-Cut for nonmembership function of $\bar{R}(t)$ is given by

$$
\bar{R}_{\beta}(t)=\left[r_{\beta}^{L}(t), r_{\beta}^{R}(t)\right]
$$

where

$$
\begin{array}{ll}
r_{\beta}^{L}(t)=\min \left(e^{-x t}\right) & \text { s.t. } \lambda_{\beta}^{L} \leq x \leq \lambda_{\beta}^{R} \\
r_{\beta}^{R}(t)=\max \left(e^{-x t}\right) & \text { s.t. } \lambda_{\beta}^{L} \leq x \leq \lambda_{\beta}^{R}
\end{array}
$$

For membership and nonmembership function, $\alpha$-Cut and $\beta$-Cut of $\tilde{R}(t)$ respectively, is

$$
\begin{aligned}
& \bar{R}_{\alpha}(t)=\left[e^{-\lambda_{\alpha}^{R} \cdot t}, e^{-\lambda_{\alpha}^{L} \cdot t}\right] \\
& \bar{R}_{\beta}(t)=\left[e^{-\lambda_{\beta}^{R} \cdot t}, e^{-\lambda_{\beta}^{L} \cdot t}\right]
\end{aligned}
$$




\subsection{Fuzzy reliability of series system using different types of conflicting bifuzzy failure rates}

Consider a system having $n$ components connected in series (Figure 10.1). Let failure rates of $n$ components are represented by different CBFN $\tilde{\lambda}_{1}, \tilde{\lambda}_{2}, \ldots, \tilde{\lambda}_{n}$ and reliability with conflicting bifuzzy failure rates $\tilde{\lambda}_{1}, \tilde{\lambda}_{2}, \ldots, \tilde{\lambda}_{n}$ are $\tilde{R}_{1}, \tilde{R}_{2}, \ldots, \tilde{R}_{n}$, respectively, at time $t$.

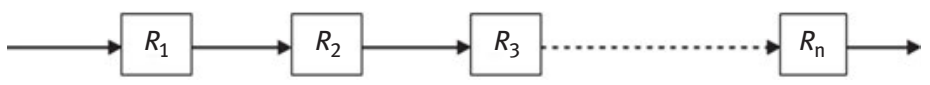

Figure 10.1: Series system.

Reliability of series system at time $t$ is

$$
\bar{R}_{S}(t)=\bar{R}_{1}(t) \otimes \bar{R}_{2}(t) \otimes \cdots \otimes \bar{R}_{n}(t)
$$

From eq. (10.2), we get

$$
\bar{R}_{i}(t)=e^{-\bar{\lambda}_{i} \cdot t}, i=1,2,3, \ldots, n ; t>0
$$

The reliability of series system $\bar{R}_{S}(t)$ is estimated as

$$
\bar{R}_{S}(t)=e^{-t \sum_{J=1}^{n} \bar{\lambda}_{J}}=e^{-\bar{\lambda}_{S} \cdot t}
$$

where $\bar{\lambda}_{s}=\psi\left(\bar{\lambda}_{1}, \bar{\lambda}_{2}, \ldots, \bar{\lambda}_{n}\right)=\sum_{j=1}^{n} \bar{\lambda}_{j}$ is failure rate.

$\alpha$-Cut of $\bar{\lambda}_{s}$ for membership function is

$$
\tilde{\lambda}_{j \alpha}=\left[h_{j \alpha}^{L}, h_{j \alpha}^{R}\right] \quad \forall \alpha \in[0,1], j=1,2, \ldots, n
$$

We have $n$ intervals $\tilde{\lambda}_{1 \alpha}=\left[h_{1 \alpha}^{L}, h_{1 \alpha}^{R}\right], \tilde{\lambda}_{2 \alpha}=\left[h_{2 \alpha}^{L}, h_{2 \alpha}^{R}\right], \ldots, \tilde{\lambda}_{1 \alpha}=\left[h_{n \alpha}^{L}, h_{n \alpha}^{R}\right] \forall \tilde{\lambda}_{j}, 1 \leq j \leq n$, respectively, for every $\alpha$.

$\beta$-Cut of nonmembership function for $\tilde{\lambda}_{S}$ is

$$
\tilde{\lambda}_{j \beta}=\left[h_{j \beta}^{L}, h_{j \beta}^{R}\right] \quad \forall \beta \in[0,1], j=1,2, \ldots, n
$$

Similarly, we have $n$ intervals $\tilde{\lambda}_{1 \beta}=\left[h_{1 \beta}^{L}, h_{1 \beta}^{R}\right], \tilde{\lambda}_{2 \beta}=\left[h_{2 \beta}^{L}, h_{2 \beta}^{R}\right], \ldots, \tilde{\lambda}_{1 \beta}=\left[h_{n \beta}^{L}, h_{n \beta}^{R}\right] \forall \tilde{\lambda}_{j}$, $1 \leq j \leq n$, respectively, for every $\beta$.

$\alpha$-Cut of $\tilde{\lambda}_{S}$ for membership function is

$$
\tilde{\lambda}_{S \alpha}=\left[\psi_{S \alpha}^{L}, \psi_{S \alpha}^{R}\right] \quad \forall \alpha \in[0,1]
$$


where

$$
\begin{gathered}
\psi_{S \alpha}^{L}=\min \sum_{j=1}^{n} x_{j}, \quad \psi_{S \alpha}^{R}=\max \sum_{j=1}^{n} x_{j} \\
\text { s.t. } h_{1 \alpha}^{L} \leq x_{1} \leq h_{1 \alpha}^{R}, h_{2 \alpha}^{L} \leq x_{2} \leq h_{2 \alpha}^{R}, \ldots, h_{n \alpha}^{L} \leq x_{n} \leq h_{n \alpha}^{R}
\end{gathered}
$$

$\beta$-Cut of $\bar{\lambda}_{s}$ for nonmembership function is

$$
\tilde{\lambda}_{S \beta}=\left[\psi_{S \beta}^{L}, \psi_{S \beta}^{R}\right] \quad \forall \beta \in[0,1]
$$

where

$$
\begin{gathered}
\psi_{S \beta}^{L}=\min \sum_{j=1}^{n} x_{j}, \psi_{S \beta}^{R}=\max \sum_{j=1}^{n} x_{j} \\
\text { s.t. } h_{1 \beta}^{L} \leq x_{1} \leq h_{1 \beta}^{R}, h_{1 \beta}^{L} \leq x_{1} \leq h_{1 \beta}^{R}, \ldots, h_{1 \beta}^{L} \leq x_{1} \leq h_{1 \beta}^{R}
\end{gathered}
$$

After solving (10.9) and (10.10), we find $\alpha$-Cut and $\beta$-Cut of fuzzy system reliability. $\alpha$-Cut and $\beta$-Cut of membership function of $\bar{R}_{s}(t)$ as

$$
\begin{aligned}
& \tilde{R}_{S \alpha}(t)=\left[e^{-\psi_{S \alpha}^{R} \cdot t}, e^{-\psi_{S \alpha}^{L} \cdot t}\right] \\
& \tilde{R}_{S \beta}(t)=\left[e^{-\psi_{S \beta}^{R} \cdot t}, e^{-\psi_{S \beta}^{L} \cdot{ }^{t}}\right]
\end{aligned}
$$

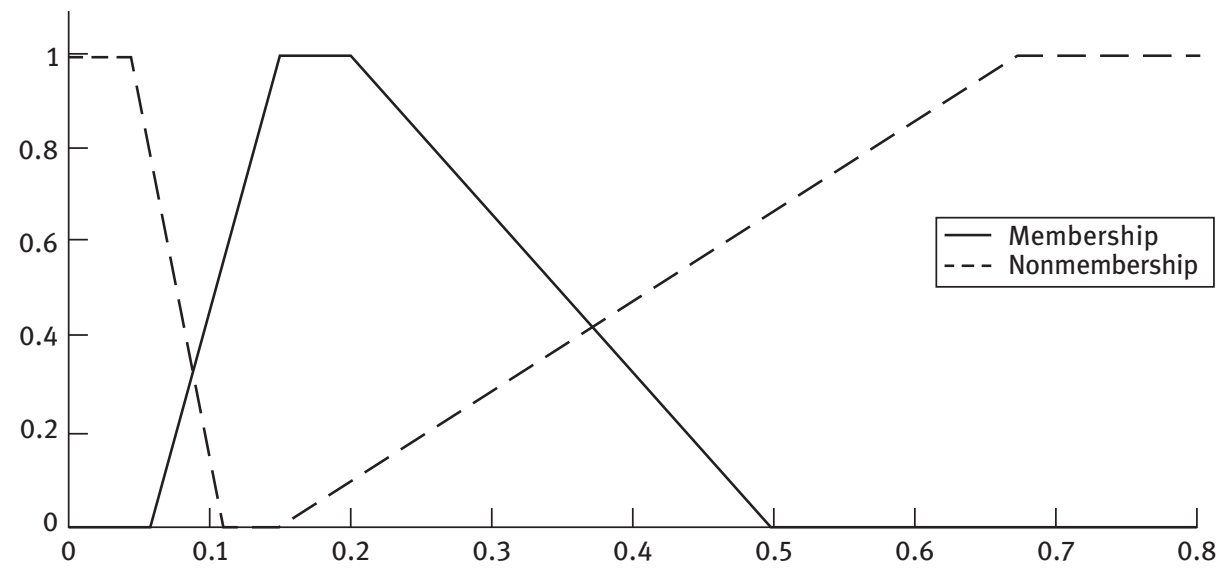

Figure 10.2: For $t=10$, membership and nonmembership function of reliability of radio set. 


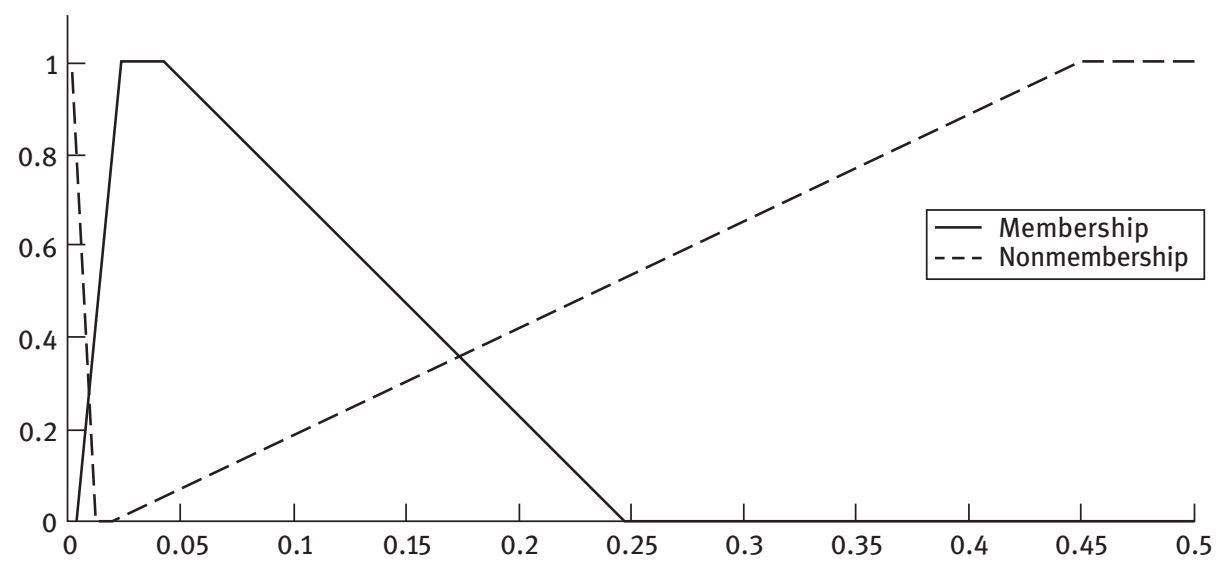

Figure 10.3: For $t=20$, membership and nonmembership function of reliability of radio set.

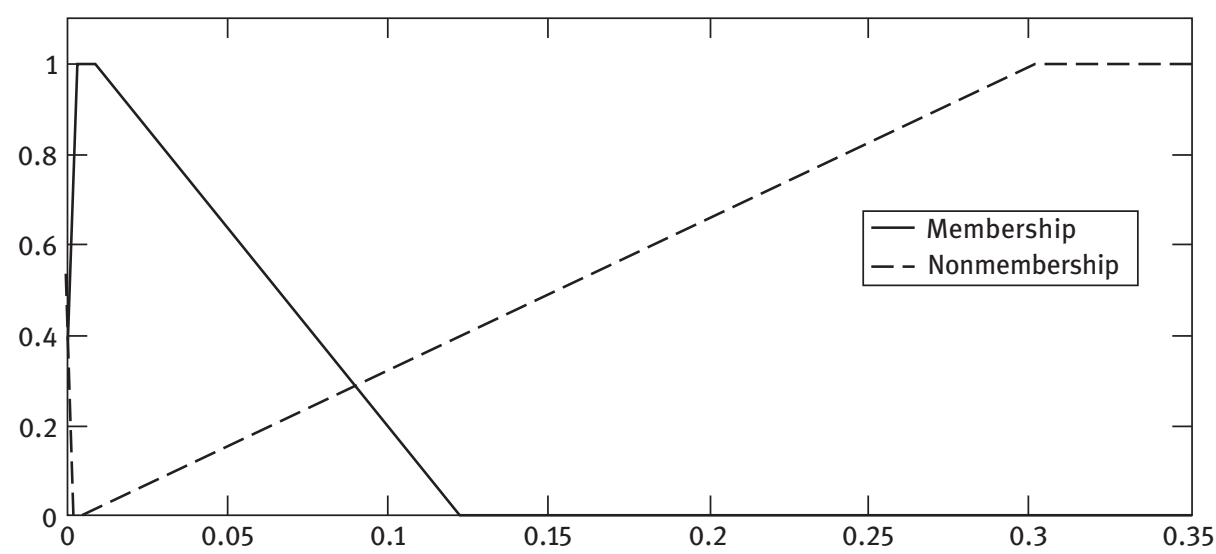

Figure 10.4: For $t=30$, membership and nonmembership function of reliability of radio set.

\subsubsection{Numerical problem}

Consider a system consisting of three independent components and all components work properly to operate the system successfully. Let failure rates of components are in the form of different types of CBFS $\tilde{\lambda}_{1}, \tilde{\lambda}_{2}$, and $\tilde{\lambda}_{3}$, respectively, assumed values of $\tilde{\lambda}_{1}, \tilde{\lambda}_{2}, \tilde{\lambda}_{3}$ are given in Table 10.1 . 
Table 10.1: Conflicting bifuzzy failure rates of components.

\begin{tabular}{ll}
\hline Bifuzzy failure rate of $i$ th component & Types of bifuzzy number \\
\hline$\tilde{\lambda}_{1}=(0.02,0.05,0.08 ; 0.01,0.05,0.09)$ & Triangular conflicting bifuzzy number \\
\hline$\tilde{\lambda}_{2}=(0.03,0.06,0.08 ; 0.02,0.07,0.09)$ & Triangular conflicting bifuzzy number \\
\hline$\tilde{\lambda}_{3}=(0.02,0.05,0.08,0.12 ; 0.01,0.07,0.10,0.13)$ & Trapezoidal conflicting bifuzzy number \\
\hline
\end{tabular}

$\alpha$-Cuts of $\tilde{\lambda}_{1}, \tilde{\lambda}_{2}, \tilde{\lambda}_{3}$ are estimated and tabulated in Table 10.2.

Table 10.2: $\alpha$-Cut of $\tilde{\lambda}_{1}, \tilde{\lambda}_{2}$ and $\tilde{\lambda}_{3}$ for membership.

\begin{tabular}{llll}
\hline$\alpha$ & $\tilde{\lambda}_{1 \alpha}$ & $\tilde{\lambda}_{2 \alpha}$ & $\tilde{\lambda}_{3 \alpha}$ \\
\hline 0.0 & {$[0.02,0.08]$} & {$[0.03,0.08]$} & {$[0.02,0.12]$} \\
\hline 0.1 & {$[0.023,0.077]$} & {$[0.033,0.078]$} & {$[0.023,0.116]$} \\
\hline 0.2 & {$[0.026,0.074]$} & {$[0.036,0.076]$} & {$[0.026,0.112]$} \\
\hline 0.3 & {$[0.029,0.071]$} & {$[0.039,0.074]$} & {$[0.029,0.108]$} \\
\hline 0.4 & {$[0.032,0.068]$} & {$[0.042,0.072]$} & {$[0.032,0.104]$} \\
\hline 0.5 & {$[0.035,0.065]$} & {$[0.045,0.070]$} & {$[0.035,0.100]$} \\
\hline 0.6 & {$[0.038,0.062]$} & {$[0.048,0.068]$} & {$[0.038,0.096]$} \\
\hline 0.7 & {$[0.041,0.059]$} & {$[0.051,0.066]$} & {$[0.041,0.092]$} \\
\hline 0.8 & {$[0.044,0.056]$} & {$[0.054,0.064]$} & {$[0.044,0.088]$} \\
\hline 0.9 & {$[0.047,0.053]$} & {$[0.057,0.062]$} & {$[0.047,0.084]$} \\
\hline 1.0 & {$[0.05,0.05]$} & {$[0.06,0.06]$} & {$[0.05,0.08]$} \\
\hline
\end{tabular}

$\beta$-Cuts of $\tilde{\lambda}_{1}, \tilde{\lambda}_{2}, \tilde{\lambda}_{3}$ are estimated and tabulated in Table 10.3.

Table 10.3: $\beta$-Cut of $\tilde{\lambda}_{1}, \tilde{\lambda}_{2}$, and $\tilde{\lambda}_{3}$ for nonmembership.

\begin{tabular}{llll}
\hline $\boldsymbol{\beta}$ & $\tilde{\lambda}_{1 \beta}$ & $\tilde{\lambda}_{2 \beta}$ & $\tilde{\lambda}_{3 \beta}$ \\
\hline 0.0 & {$[0.05,0.05]$} & {$[0.07,0.07]$} & {$[0.07,0.10]$} \\
\hline 0.1 & {$[0.046,0.054]$} & {$[0.065,0.072]$} & {$[0.064,0.103]$} \\
\hline 0.2 & {$[0.042,0.058]$} & {$[0.060,0.074]$} & {$[0.058,0.106]$} \\
\hline 0.3 & {$[0.038,0.062]$} & {$[0.055,0.076]$} & {$[0.052,0.109]$} \\
\hline 0.4 & {$[0.034,0.066]$} & {$[0.050,0.078]$} & {$[0.046,0.112]$} \\
\hline
\end{tabular}


Table 10.3 (continued)

\begin{tabular}{llll}
\hline $\boldsymbol{\beta}$ & $\tilde{\lambda}_{1 \beta}$ & $\tilde{\lambda}_{2 \beta}$ & $\tilde{\lambda}_{3 \beta}$ \\
\hline 0.5 & {$[0.03,0.07]$} & {$[0.045,0.080]$} & {$[0.040,0.115]$} \\
\hline 0.6 & {$[0.026,0.074]$} & {$[0.040,0.082]$} & {$[0.034,0.118]$} \\
\hline 0.7 & {$[0.022,0.078]$} & {$[0.035,0.084]$} & {$[0.028,0.121]$} \\
\hline 0.8 & {$[0.018,0.082]$} & {$[0.030,0.086]$} & {$[0.022,0.124]$} \\
\hline 0.9 & {$[0.014,0.086]$} & {$[0.025,0.088]$} & {$[0.016,0.127]$} \\
\hline 1.0 & {$[0.01,0.09]$} & {$[0.02,0.09]$} & {$[0.01,0.13]$} \\
\hline
\end{tabular}

Using formulas (10.9) to (10.11), we get fuzzy reliability of series system

$$
\begin{aligned}
\tilde{R}_{S \alpha}(t) & =\left[e^{-(.28-.09 \alpha) t}, e^{-(.07+.09 \alpha) t}\right] \\
\tilde{R}_{S \beta}(t) & =\left[e^{-(.13+.09 \beta) t}, e^{-(.19-.15 \beta) t}\right]
\end{aligned}
$$

The fuzzy system reliability of given system is calculated by eq. (10.12). $\alpha$-Cut and $\beta$-Cut of fuzzy reliability are computed for $t=10,20,30$ shown in Table 10.4 and fig. 10.2, $10.3 \&$ 10.4. The fuzzy reliability of above system is in trapezoidal CBFN for failure rates as conflicting bifuzzy of components for different time $t$.

\subsection{Fuzzy reliability of parallel system using of conflicting bifuzzy failure rates}

Consider a system consisting " $n$ " components are in parallel form (Figure 10.5). Let us consider failure rates of $n$ components are in form of different CBFNs $\tilde{\lambda}_{1}, \tilde{\lambda}_{2}, \ldots, \tilde{\lambda}_{n}$. Let the fuzzy reliability of $n$ components with conflicting bifuzzy failure rates are $\tilde{\lambda}_{1}, \tilde{\lambda}_{2}, \ldots, \tilde{\lambda}_{n}$ and $\tilde{R}_{1}, \tilde{R}_{2}, \ldots, \tilde{R}_{n}$, respectively, at time $t$.

Reliability of parallel system at time $t$ is

$$
\tilde{R}_{P}(t)=1-\prod_{i=1}^{n}\left(1-\tilde{R}_{i}(t)\right)=1-\prod_{i=1}^{n}\left(1-\exp \left(-\tilde{\lambda}_{i} \cdot t\right)\right)
$$

So $\tilde{R}_{P}(t)=\psi\left(\tilde{\lambda}_{1}, \tilde{\lambda}_{2}, \ldots, \tilde{\lambda}_{n} ; t\right)=1-\prod_{i=1}^{n}\left(1-\exp \left(-\tilde{\lambda}_{i} . t\right)\right)$

$\alpha$-Cut of $\tilde{\lambda}_{i}$ for membership function is

$$
\tilde{\lambda}_{i \alpha}=\left[h_{i \alpha}^{L}, h_{i \alpha}^{R}\right] \quad \forall \alpha \in[0,1], i=1,2,3, \ldots, n
$$

We have $n$ intervals $\tilde{\lambda}_{1 \alpha}=\left[h_{1 \alpha}^{L}, h_{1 \alpha}^{R}\right], \tilde{\lambda}_{2 \alpha}=\left[h_{2 \alpha}^{L}, h_{2 \alpha}^{R}\right], \ldots, \tilde{\lambda}_{n \alpha}=\left[h_{n \alpha}^{L}, h_{n \alpha}^{R}\right] \forall \tilde{\lambda}_{j}, 1 \leq j \leq n$ respectively, for each $\alpha$. 


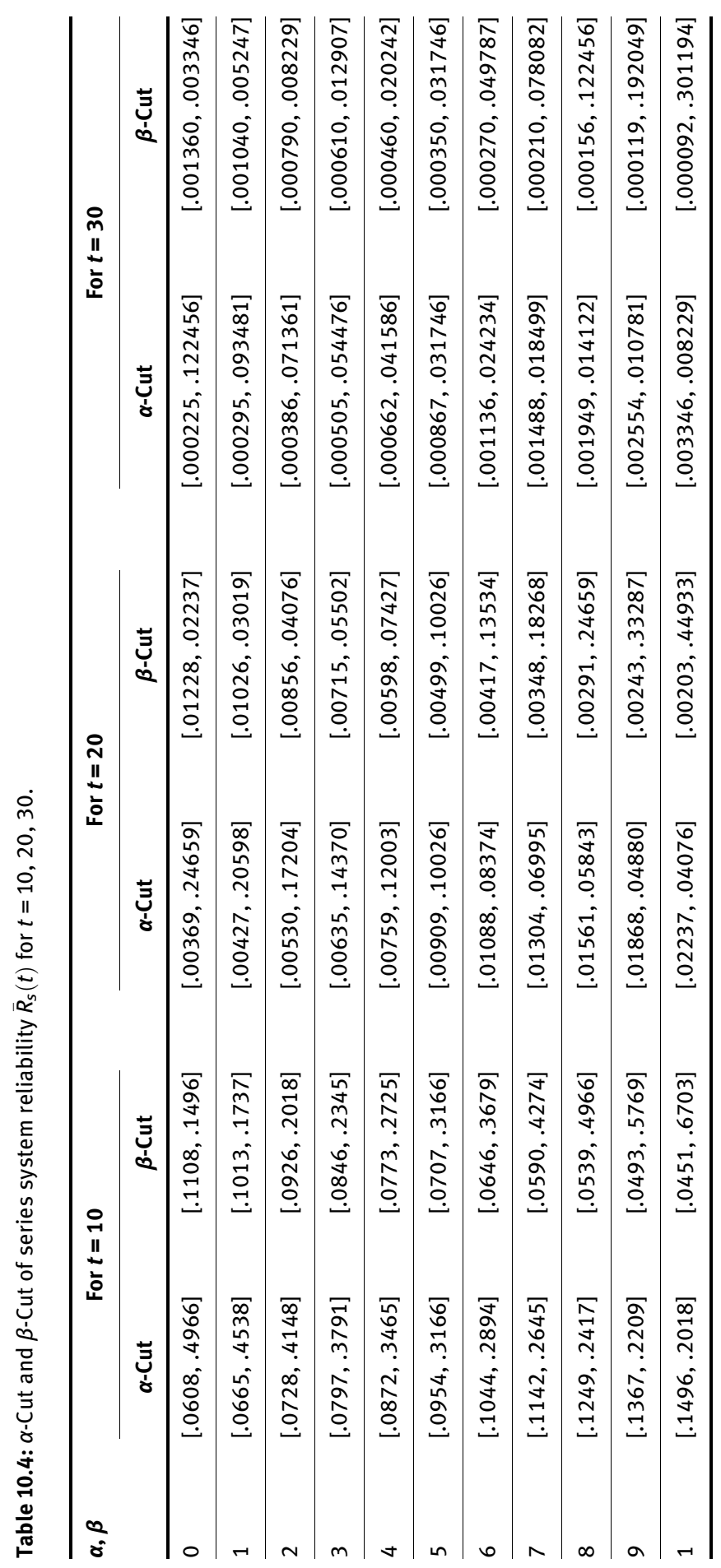




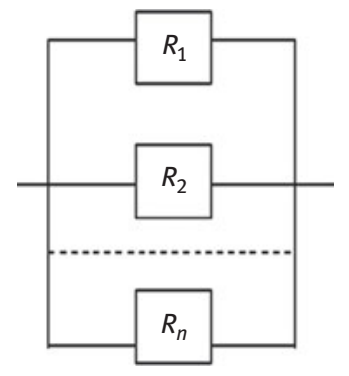

Figure 10.5: Parallel system.

$\beta$-Cut of nonmembership function of $\tilde{\lambda}_{i}$ is

$$
\tilde{\lambda}_{i \beta}=\left[h_{i \beta}^{L}, h_{i \beta}^{R}\right] \quad \forall \beta \in[0,1], i=1,2,3, \ldots, n .
$$

Similarly, we have $n$ intervals $\tilde{\lambda}_{1 \beta}=\left[h_{1 \beta}^{L}, h_{1 \beta}^{R}\right], \tilde{\lambda}_{2 \beta}=\left[h_{2 \beta}^{L}, h_{2 \beta}^{R}\right], \ldots, \tilde{\lambda}_{n \beta}=\left[h_{n \beta}^{L}, h_{n \beta}^{R}\right] \forall \tilde{\lambda}_{j}$, $1 \leq j \leq n$, respectively, for each $\beta$.

$\alpha$-Cut of $\tilde{R}_{P}(t)$ for membership function is

$$
\tilde{R}_{P \alpha}=\left[\psi_{P \alpha}^{L}(t), \psi_{P \alpha}^{R}(t)\right]
$$

where

$$
\begin{aligned}
& \psi_{P \alpha}^{L}(t)=\min \left(1-\prod_{j=1}^{n}\left(1-e^{-x_{J} \cdot t}\right)\right), \psi_{P \alpha}^{L}(t)=\max \left(1-\prod_{j=1}^{n}\left(1-e^{-x_{J} \cdot t}\right)\right) \\
& \text { s.t. } h_{1 \alpha}^{L} \leq x_{1} \leq h_{1 \alpha}^{R}, h_{2 \alpha}^{L} \leq x_{2} \leq h_{2 \alpha}^{R}, \ldots, h_{n \alpha}^{L} \leq x_{n} \leq h_{n \alpha}^{R} .
\end{aligned}
$$

$\beta$-Cut of nonmembership function of $\bar{R}_{P}(t)$ is as

$$
\tilde{R}_{P \beta}=\left[\psi_{P \beta}^{L}(t), \psi_{P \beta}^{R}(t)\right]
$$

where

$$
\begin{aligned}
& \psi_{P \beta}^{L}(t)=\min \left(1-\prod_{i=1}^{n}\left(1-e^{-x_{i} \cdot t}\right)\right), \quad \psi_{P \beta}^{R}(t)=\max \left(1-\prod_{i=1}^{n}\left(1-e^{-x_{i} \cdot t}\right)\right) \\
& \text { s.t. } h_{1 \beta}^{L} \leq x_{1} \leq h_{1 \beta}^{R}, h_{1 \beta}^{L} \leq x_{1} \leq h_{1 \beta}^{R}, \ldots, h_{1 \beta}^{L} \leq x_{1} \leq h_{1 \beta}^{R} .
\end{aligned}
$$

After solving eqs. (10.14) and (10.15), we find $\alpha$-Cut and $\beta$-Cut of fuzzy reliability of parallel system $\bar{R}_{P}(t)$. 


\subsubsection{Numerical problem}

Consider a system has three independent components. At least one of them must work properly to operate given system properly. Let conflicting bifuzzy failure rates of components are in the form of CBFNs $\tilde{\lambda}_{1}, \tilde{\lambda}_{2}$, and $\tilde{\lambda}_{3}$, respectively. For numerical computation assumed values of $\tilde{\lambda}_{1}, \tilde{\lambda}_{2}$, and $\tilde{\lambda}_{3}$ are given in Table 10.5.

Table 10.5: Conflicting bifuzzy failure rates of components.

\begin{tabular}{ll}
\hline Bifuzzy failure rate & Types of bifuzzy number \\
\hline$\tilde{\lambda}_{1}=(0.3,0.4,0.5 ; 0.2,0.4,0.6)$ & Triangular conflicting bifuzzy number \\
\hline$\tilde{\lambda}_{2}=(0.2,0.5,0.7 ; 0.3,0.6,0.8)$ & Triangular conflicting bifuzzy number \\
\hline$\tilde{\lambda}_{3}=(0.03,0.05,0.08,0.10 ; 0.02,0.06,0.10,0.12)$ & Trapezoidal conflicting bifuzzy number \\
\hline
\end{tabular}

$\alpha$-Cuts of $\tilde{\lambda}_{1}, \tilde{\lambda}_{2}, \tilde{\lambda}_{3}$ are evaluated and given in Table 10.6.

Table 10.6: For membership, $\alpha$-Cut of $\tilde{\lambda}_{1}, \tilde{\lambda}_{2}$, and $\tilde{\lambda}_{3}$.

\begin{tabular}{llll}
\hline$\alpha$ & $\tilde{\lambda}_{1 \alpha}$ & $\tilde{\lambda}_{2 \alpha}$ & $\tilde{\lambda}_{3 \alpha}$ \\
\hline 0.0 & {$[0.3,0.5]$} & {$[0.2,0.7]$} & {$[0.03,0.10]$} \\
\hline 0.1 & {$[0.31,0.49]$} & {$[0.23,0.68]$} & {$[0.032,0.098]$} \\
\hline 0.2 & {$[0.32,0.48]$} & {$[0.26,0.66]$} & {$[0.034,0.096]$} \\
\hline 0.3 & {$[0.33,0.47]$} & {$[0.29,0.64]$} & {$[0.036,0.094]$} \\
\hline 0.4 & {$[0.34,0.46]$} & {$[0.32,0.62]$} & {$[0.038,0.092]$} \\
\hline 0.5 & {$[0.35,0.45]$} & {$[0.35,0.60]$} & {$[0.04,0.09]$} \\
\hline 0.6 & {$[0.36,0.44]$} & {$[0.38,0.58]$} & {$[0.042,0.088]$} \\
\hline 0.7 & {$[0.37,0.43]$} & {$[0.41,0.56]$} & {$[0.044,0.086]$} \\
\hline 0.8 & {$[0.38,0.42]$} & {$[0.44,0.54]$} & {$[0.046,0.084]$} \\
\hline 0.9 & {$[0.39,0.41]$} & {$[0.47,0.52]$} & {$[0.048,0.082]$} \\
\hline 1.0 & {$[0.4,0.4]$} & {$[0.5,0.5]$} & {$[0.05,0.08]$} \\
\hline
\end{tabular}


$\beta$-Cut of $\tilde{\lambda}_{1}, \tilde{\lambda}_{2}$, and $\tilde{\lambda}_{3}$ are evaluated and given in Table 10.7.

Table 10.7: For nonmembership, $\beta$-Cut of $\tilde{\lambda}_{1}, \tilde{\lambda}_{2}$, and $\tilde{\lambda}_{3}$.

\begin{tabular}{llll}
\hline$\beta$ & $\tilde{\lambda}_{1 \beta}$ & $\tilde{\lambda}_{2 \beta}$ & $\tilde{\lambda}_{3 \beta}$ \\
\hline 0.0 & {$[0.4,0.4]$} & {$[0.6,0.6]$} & {$[0.06,0.10]$} \\
\hline 0.1 & {$[0.38,0.42]$} & {$[0.57,0.62]$} & {$[0.056,0.102]$} \\
\hline 0.2 & {$[0.36,0.44]$} & {$[0.54,0.64]$} & {$[0.052,0.104]$} \\
\hline 0.3 & {$[0.34,0.46]$} & {$[0.51,0.66]$} & {$[0.048,0.106]$} \\
\hline 0.4 & {$[0.32,0.48]$} & {$[0.48,0.68]$} & {$[0.044,0.108]$} \\
\hline 0.5 & {$[0.3,0.5]$} & {$[0.45,0.70]$} & {$[0.04,0.11]$} \\
\hline 0.6 & {$[0.28,0.52]$} & {$[0.42,0.72]$} & {$[0.036,0.112]$} \\
\hline 0.7 & {$[0.26,0.54]$} & {$[0.39,0.74]$} & {$[0.032,0.114]$} \\
\hline 0.8 & {$[0.24,0.56]$} & {$[0.36,0.76]$} & {$[0.028,0.116]$} \\
\hline 0.9 & {$[0.22,0.58]$} & {$[0.33,0.78]$} & {$[0.024,0.118]$} \\
\hline 1.0 & {$[0.2,0.6]$} & {$[0.3,0.8]$} & {$[0.02,0.12]$} \\
\hline
\end{tabular}

Using formulas (10.14) to (10.15), we get

$$
\begin{aligned}
& \psi_{P \alpha}^{L}(t)=1-\left(1-e^{-(.5-.1 \alpha) t}\right) \times\left(1-e^{-(.7-.2 \alpha) t}\right) \times\left(1-e^{-(.1-.02 \alpha) t}\right) \\
& \psi_{P \alpha}^{R}(t)=1-\left(1-e^{-(.3+.1 \alpha) t}\right) \times\left(1-e^{-(.2+.3 \alpha) t}\right) \times\left(1-e^{-(.03+.02 \alpha) t}\right) \\
& \psi_{P \beta}^{L}(t)=1-\left(1-e^{-(.4+.2 \beta) t}\right) \times\left(1-e^{-(.6+.2 \beta) t}\right) \times\left(1-e^{-(.1+.02 \beta) t}\right) \\
& \psi_{P \beta}^{R}(t)=1-\left(1-e^{-(.4-.2 \beta) t}\right) \times\left(1-e^{-(.6-.3 \beta) t}\right) \times\left(1-e^{-(.06-.04 \beta) t}\right)
\end{aligned}
$$

The system reliability of given system is evaluated using (10.16). $\alpha$-Cut and $\beta$-Cut of fuzzy reliability of given parallel system are computed for $t=10,20,30$ and are tabulated in Table 10.8 and Fig. 10.6, $10.7 \& 10.8$. The fuzzy reliability of system is in trapezoidal CBFN.

\subsection{Reliability of parallel-series system when failure rates follow conflicting bifuzzy numbers}

Let us consider a parallel-series system consisting “ $j$ ” branches connected in parallel form and each branch contains “” components connected in series form as 


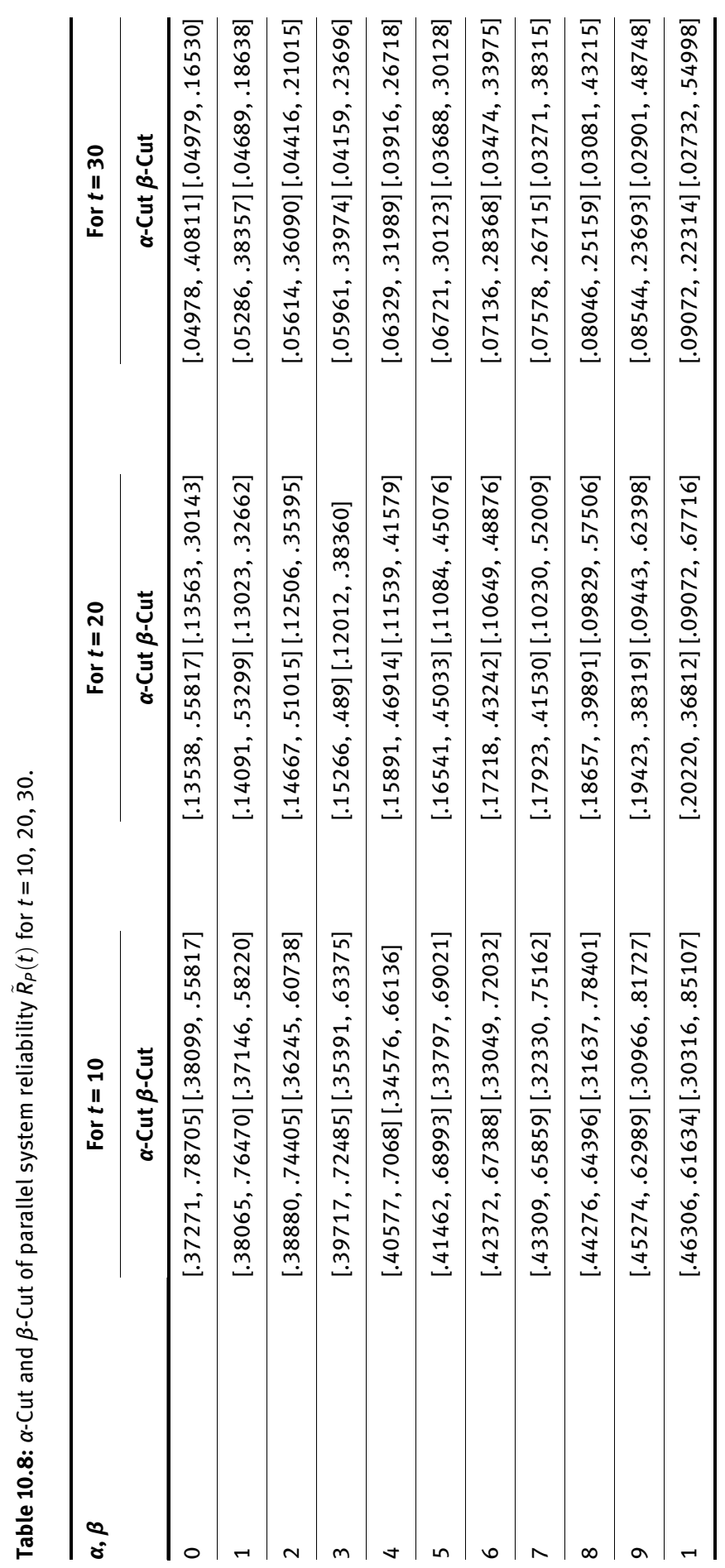




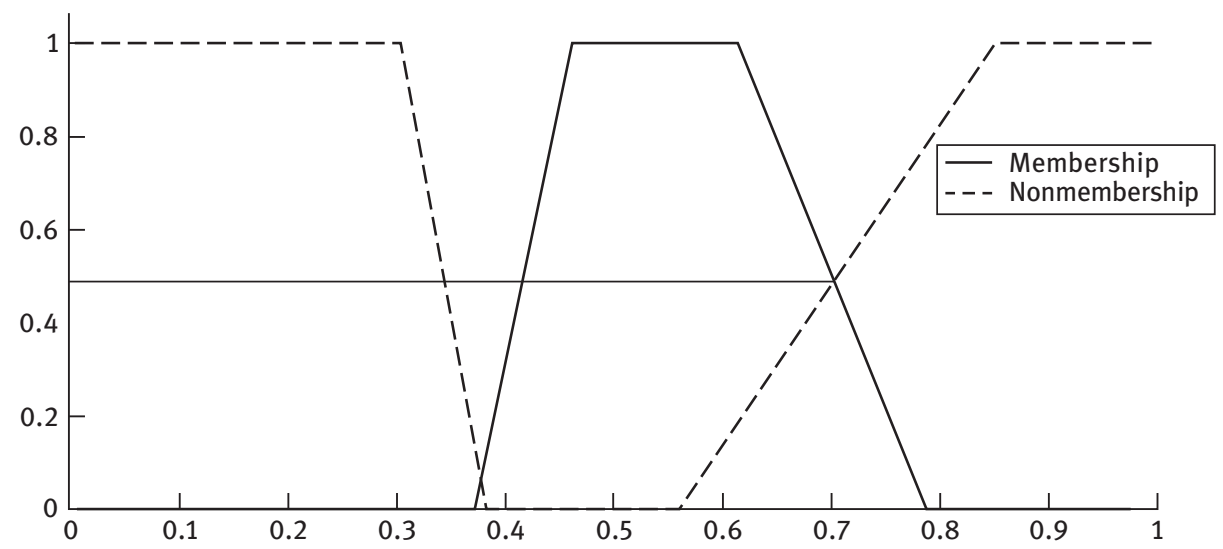

Figure 10.6: For $t=10$, membership function and nonmembership function of reliability of achieving orbit.

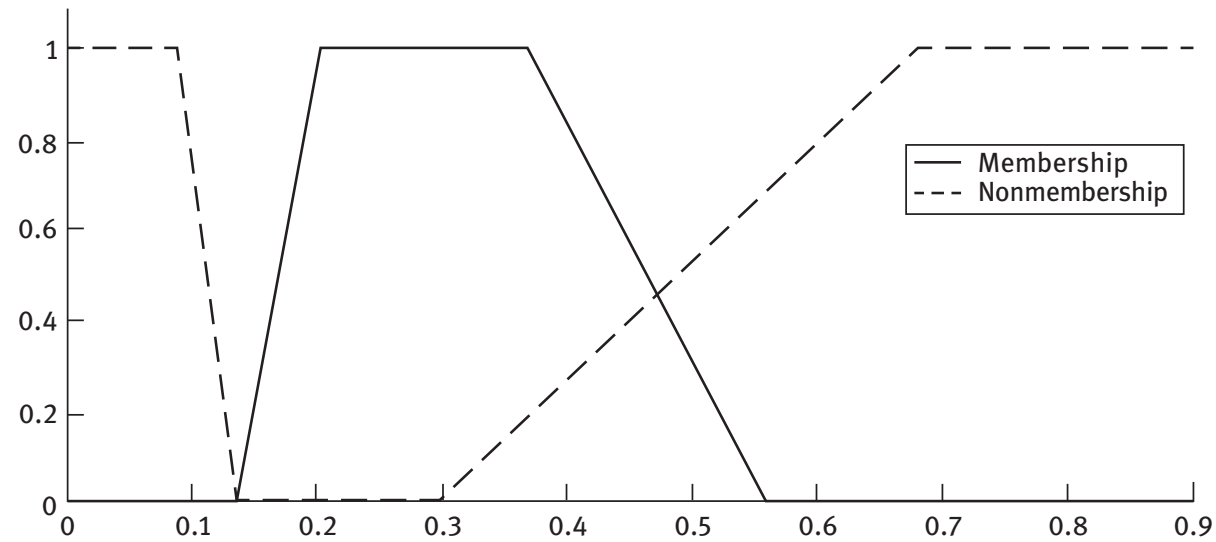

Figure 10.7: For $t=20$, membership function and nonmembership function of reliability of achieving orbit.

shown in Figure 10.9. Let us consider failure rates of all components are following different CBFNs $\tilde{\lambda}_{r s}$.

Let failure rate function of the $s$ th component of $r$ th branch $(r=1,2, \ldots, i$ and $s=1,2, \ldots, j$ ) is represented by CBFS $\tilde{\lambda}_{r s}$.

Reliability of the $s$ th component of $r$ th branch is as follows:

$$
\tilde{R}_{r s}(t)=\exp \left(-\tilde{\lambda}_{r s} . t\right)
$$

It is well known that the fuzzy reliability of system $\bar{R}_{P S}(t)$ is given by 


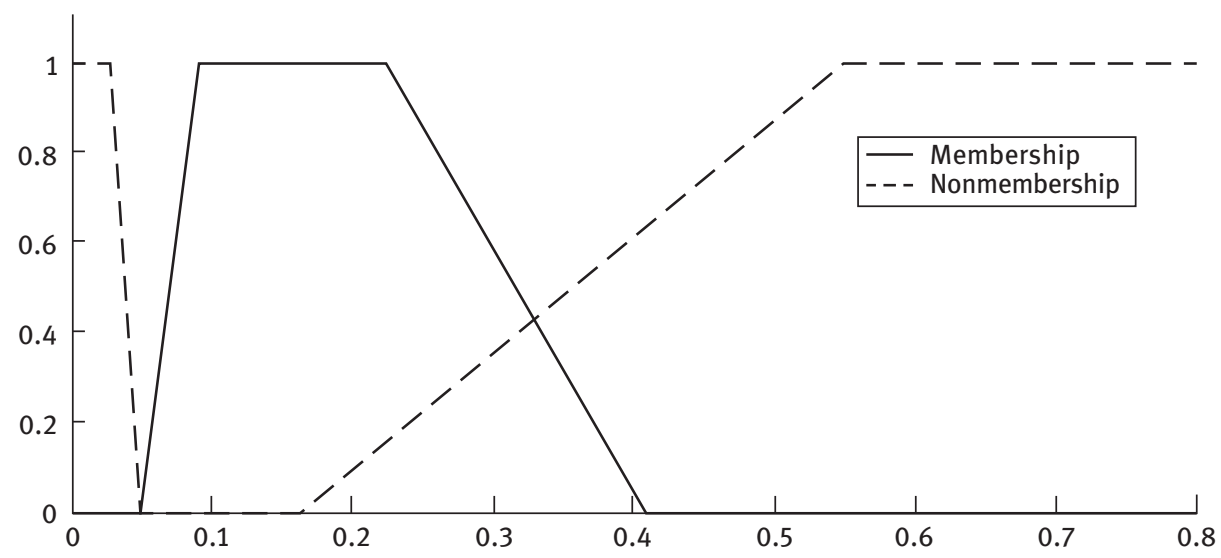

Figure 10.8: For $t=30$, membership function and nonmembership function of reliability of achieving orbit.

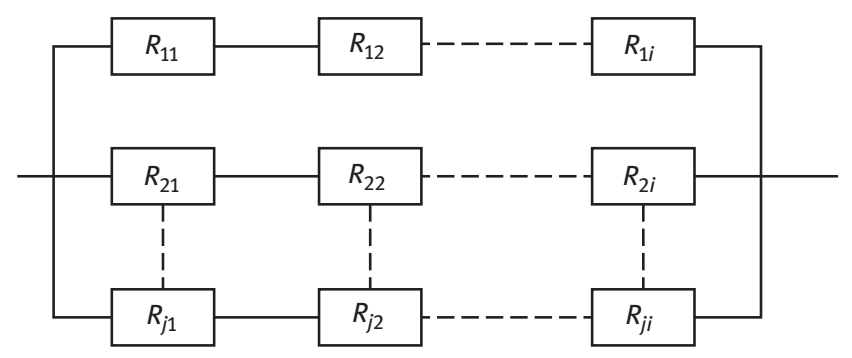

Figure 10.9: Parallel-series system.

$$
\bar{R}_{P S}(t)=1-\prod_{s=1}^{j}\left(1-\prod_{r=1}^{i} \tilde{R}_{r s}\right)=1-\prod_{s=1}^{j}\left(1-\prod_{r=1}^{i} \exp \left(-\tilde{\lambda}_{r s} . t\right)\right)
$$

So $\bar{R}_{P S}(t)=\psi\left(\tilde{\lambda}_{r s}: r=1,2, \ldots, i\right.$ and $s=1,2, \ldots, j ;=1-\prod_{s=1}^{j}\left(1-\prod_{r=1}^{i} \exp \left(-\tilde{\lambda}_{r s} . t\right)\right)$
$\alpha$-Cut of $\tilde{\lambda}_{r s}$ for membership function is

$$
\tilde{\lambda}_{r s \alpha}=\left[h_{r s \alpha}^{L}, h_{r s \alpha}^{R}\right] \quad \forall \alpha \in[0,1], r=1,2, \ldots, i \text { and } s=1,2, \ldots, j
$$

For each $\alpha$, we have $n^{2}$ intervals $\tilde{\lambda}_{11 \alpha}=\left[h_{11 \alpha}^{L}, h_{11 \alpha}^{R}\right], \tilde{\lambda}_{12 \alpha}=\left[h_{12 \alpha}^{L}, h_{12 \alpha}^{R}\right], \tilde{\lambda}_{21 \alpha}=\left[h_{21 \alpha}^{L}, h_{21 \alpha}^{R}\right]$ $, \ldots, \tilde{\lambda}_{i j \alpha}=\left[h_{i j \alpha}^{L}, h_{i j \alpha}^{R}\right]$, for each $\tilde{\lambda}_{r s}, 1 \leq r \leq i$ and $1 \leq s \leq j$, respectively.

For nonmembership function, $\beta$-Cut of $\tilde{\lambda}_{r s}$ is

$$
\tilde{\lambda}_{r s \beta}=\left[h_{r s \beta}^{L}, h_{r s \beta}^{R}\right] \quad \forall \beta \in[0,1], r=1,2, \ldots, i \text { and } s=1,2, \ldots, j
$$

Similarly, for every $\beta$, we have $n^{2}$ intervals $\tilde{\lambda}_{11 \beta}=\left[h_{11 \beta}^{L}, h_{11 \beta}^{R}\right], \tilde{\lambda}_{12 \beta}=\left[h_{12 \beta}^{L}, h_{12 \beta}^{R}\right]$, $\tilde{\lambda}_{21 \beta}=\left[h_{21 \beta}^{L}, h_{21 \beta}^{R}\right], \ldots, \tilde{\lambda}_{i j \beta}=\left[h_{i j \beta}^{L}, h_{i j \beta}^{R}\right]$, for each $\tilde{\lambda}_{r s}, 1 \leq r \leq i$ and $1 \leq s \leq j$, respectively. 
$\alpha$-Cut of $\tilde{R}_{P S}(t)$ for membership function is

$$
\tilde{R}_{P S \alpha}(t)=\left[\psi_{P S \alpha}^{L}(t), \psi_{P S \alpha}^{R}(t)\right]
$$

where

$$
\begin{aligned}
& \psi_{P S \alpha}^{L}(t)=\min \\
& \left\{1-\prod_{s=1}^{j}\left(1-\prod_{r=1}^{i} \exp \left(-\tilde{\lambda}_{r s} . t\right)\right)\right\}, \quad \psi_{P S \alpha}^{L}(t)=\min \left\{1-\prod_{s=1}^{j}\left(1-\prod_{r=1}^{i} \exp \left(-\tilde{\lambda}_{r s} . t\right)\right)\right\} \\
& \text { s.t. } h_{11 \alpha}^{L} \leq x_{11} \leq h_{11 \alpha}^{R}, h_{12 \alpha}^{L} \leq x_{12} \leq h_{12 \alpha}^{R}, h_{21 \alpha}^{L} \leq x_{21} \leq h_{21 \alpha}^{R}, \ldots, h_{i j \alpha}^{L} \leq x_{i j} \leq h_{i j \alpha}^{R}
\end{aligned}
$$

$\beta$-Cut of $\tilde{R}_{P S}(t)$ for nonmembership function is

$$
\tilde{R}_{P S \beta}(t)=\left[\psi_{P S \beta}^{L}(t), \psi_{P S \beta}^{R}(t)\right]
$$

where

$$
\begin{aligned}
& \psi_{P S \beta}^{L}(t)=\min \\
& \left\{1-\prod_{s=1}^{j}\left(1-\prod_{r=1}^{i} \exp \left(-\tilde{\lambda}_{r s} . t\right)\right)\right\}, \quad \psi_{P S \beta}^{L}(t)=\min \left\{1-\prod_{s=1}^{j}\left(1-\prod_{r=1}^{i} \exp \left(-\tilde{\lambda}_{r s} . t\right)\right)\right\} \\
& \text { s.t. } h_{11 \beta}^{L} \leq x_{11} \leq h_{11 \beta}^{R}, h_{12 \beta}^{L} \leq x_{12} \leq h_{12 \beta}^{R}, h_{21 \beta}^{L} \leq x_{21} \leq h_{21 \beta}^{R}, \ldots, h_{i j \beta}^{L} \leq x_{i j} \leq h_{i j \beta}^{R}
\end{aligned}
$$

After solving (10.18) and (10.19), we calculate $\alpha$ and $\beta$-Cut of fuzzy reliability of above system $\tilde{R}_{P S}(t)$.

\subsubsection{Numerical example}

Suppose there is a communication system which receives the input signal and transmits the output signal. For this, there are two receivers and two transmitters in the system connected as is in Figure 10.10. For a successful communication at least one receiver and one transmitter connected in series configuration must work properly.

The failure rates of receivers and transmitters are in the form of different types of CBFNs given in Table 10.9.

The $\alpha$-Cuts of $\tilde{\lambda}_{11}, \tilde{\lambda}_{12}, \tilde{\lambda}_{21}, \tilde{\lambda}_{22}$ are computed and tabulated in Table 10.10.

The $\beta$-Cut of $\tilde{\lambda}_{11}, \tilde{\lambda}_{12}, \tilde{\lambda}_{21}, \tilde{\lambda}_{22}$ are computed and tabulated in Table 10.11.

Using formulation (10.18) and (10.19), we get 


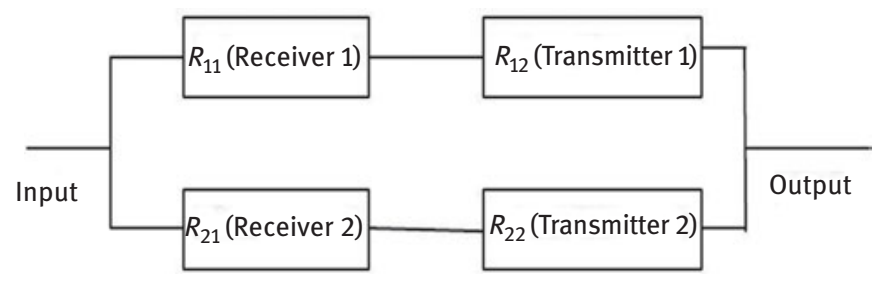

Figure 10.10: Parallel-series system.

Table 10.9: Conflicting bifuzzy failure rates of components.

\begin{tabular}{ll}
\hline Bifuzzy failure rate & Types of bifuzzy number \\
\hline$\tilde{\lambda}_{11}=(0.05,0.10,0.15 ; 0.04,0.12,0.18)$ & Triangular conflicting bifuzzy number \\
\hline$\tilde{\lambda}_{12}=(0.06,0.09,0.12,0.16 ; 0.07,0.11,0.15,0.18)$ & Trapezoidal conflicting bifuzzy number \\
$\tilde{\lambda}_{21}=(0.07,0.12,0.17 ; 0.09,0.14,0.18)$ & Triangular conflicting bifuzzy number \\
$\tilde{\lambda}_{22}=(0.03,0.08,0.13,018 ; 0.05,0.10,0.18,0.23)$ & Trapezoidal conflicting bifuzzy number \\
\hline
\end{tabular}

Table 10.10: $\alpha$-Cut of $\tilde{\lambda}_{11}, \tilde{\lambda}_{12}, \tilde{\lambda}_{21}$, and $\tilde{\lambda}_{22}$ for membership.

\begin{tabular}{lllll}
\hline$\alpha$ & $\tilde{\lambda}_{11 \alpha}$ & $\tilde{\lambda}_{12 \alpha}$ & $\tilde{\lambda}_{21 \alpha}$ & $\tilde{\lambda}_{22 \alpha}$ \\
\hline 0.0 & {$[0.05,0.15]$} & {$[0.06,0.16]$} & {$[0.07,0.17]$} & {$[0.03,0.18]$} \\
\hline 0.1 & {$[0.055,0.145]$} & {$[0.063,0.156]$} & {$[0.075,0.165]$} & {$[0.035,0.175]$} \\
\hline 0.2 & {$[0.06,0.14]$} & {$[0.066,0.152]$} & {$[0.08,0.16]$} & {$[0.04,0.17]$} \\
\hline 0.3 & {$[0.065,0.135]$} & {$[0.069,0.148]$} & {$[0.085,0.155]$} & {$[0.045,0.165]$} \\
\hline 0.4 & {$[0.07,0.13]$} & {$[0.072,0.144]$} & {$[0.09,0.15]$} & {$[0.05,0.16]$} \\
\hline 0.5 & {$[0.075,0.125]$} & {$[0.075,0.140]$} & {$[0.095,0.145]$} & {$[0.055,0.155]$} \\
\hline 0.6 & {$[0.08,0.12]$} & {$[0.078,0.136]$} & {$[0.10,0.14]$} & {$[0.06,0.15]$} \\
\hline 0.7 & {$[0.085,0.115]$} & {$[0.081,0.132]$} & {$[0.105,0.135]$} & {$[0.065,0.145]$} \\
\hline 0.8 & {$[0.09,0.11]$} & {$[0.084,0.128]$} & {$[0.11,0.13]$} & {$[0.07,0.14]$} \\
\hline 0.9 & {$[0.095,0.105]$} & {$[0.087,0.124]$} & {$[0.115,0.125]$} & {$[0.075,0.135]$} \\
\hline 1.0 & {$[0.10,0.10]$} & {$[0.09,0.12]$} & {$[0.12,0.12]$} & {$[0.08,0.13]$} \\
\hline
\end{tabular}


Table 10.11: $\beta$-Cut of $\tilde{\lambda}_{11}, \tilde{\lambda}_{12}, \tilde{\lambda}_{21}$, and $\tilde{\lambda}_{22}$ for nonmembership.

\begin{tabular}{lllll}
\hline $\boldsymbol{\beta}$ & $\tilde{\lambda}_{11 \beta}$ & $\tilde{\lambda}_{12 \beta}$ & $\tilde{\lambda}_{21 \beta}$ & $\tilde{\lambda}_{22 \alpha}$ \\
\hline 0.0 & {$[0.12,0.12]$} & {$[0.11,0.15]$} & {$[0.14,0.14]$} & {$[0.10,0.18]$} \\
\hline 0.1 & {$[0.112,0.126]$} & {$[0.106,0.153]$} & {$[0.135,0.144]$} & {$[0.095,0.185]$} \\
\hline 0.2 & {$[0.104,0.132]$} & {$[0.102,0.156]$} & {$[0.130,0.148]$} & {$[0.09,0.19]$} \\
\hline 0.3 & {$[0.096,0.138]$} & {$[0.098,0.159]$} & {$[0.125,0.152]$} & {$[0.085,0.195]$} \\
\hline 0.4 & {$[0.088,0.144]$} & {$[0.094,0.162]$} & {$[0.120,0.156]$} & {$[0.08,0.20]$} \\
\hline 0.5 & {$[0.08,0.15]$} & {$[0.090,0.165]$} & {$[0.115,0.160]$} & {$[0.075,0.205]$} \\
\hline 0.6 & {$[0.072,0.156]$} & {$[0.086,0.168]$} & {$[0.110,0.164]$} & {$[0.07,0.21]$} \\
\hline 0.7 & {$[0.064,0.162]$} & {$[0.082,0.171]$} & {$[0.105,0.168]$} & {$[0.065,0.215]$} \\
\hline 0.8 & {$[0.056,0.168]$} & {$[0.078,0.174]$} & {$[0.10,0.172]$} & {$[0.06,0.22]$} \\
\hline 0.9 & {$[0.048,0.174]$} & {$[0.074,0.177]$} & {$[0.095,0.176]$} & {$[0.055,0.225]$} \\
\hline 1.0 & {$[0.04,0.18]$} & {$[0.07,0.18]$} & {$[0.09,0.18]$} & {$[0.05,0.23]$} \\
\hline
\end{tabular}

$$
\begin{aligned}
& \psi_{P S \alpha}^{L}(t)=1-\left\{\left(1-e^{-(.36-.1 \alpha) t}\right) \times\left(1-e^{-(.34-.09 \alpha) t}\right)\right\} \\
& \psi_{P S \alpha}^{R}(t)=1-\left\{\left(1-e^{-(.12+.1 \alpha) t}\right) \times\left(1-e^{-(.09+.08 \alpha) t}\right)\right\} \\
& \psi_{P S \beta}^{L}(t)=1-\left\{\left(1-e^{-(.30+.1 \beta) t}\right) \times\left(1-e^{-(.33+.08 \beta) t}\right)\right\} \\
& \psi_{P S \beta}^{R}(t)=1-\left\{\left(1-e^{-(.26-.13 \beta) t}\right) \times\left(1-e^{-(.21-.09 \beta) t}\right)\right\}
\end{aligned}
$$

The fuzzy system reliability of given system is evaluated using (10.20). The fuzzy reliability of system is trapezoidal CBFN for failure rate of components follow conflicting bifuzzy. The $\alpha$-Cut and $\beta$-Cut of fuzzy reliability $\bar{R}_{P S}(t)$ are evaluated for $t=$ 10, 20, 30 and shown in Table 10.12. The value of sum of membership and nonmembership degree is found to be greater than one (Figures 10.11-10.13) at point $A, B, C$.

\subsection{Reliability of series-parallel system when failure rates follow conflicting bifuzzy numbers}

Let us assume a series-parallel system consisting “” subsystems connected in series and each subsystem contains " $j$ " components connected in parallel form as shown in Figure 10.14. Let failure rates of all components are assumed to be different CBFNs $\tilde{\lambda}_{r s}$. 


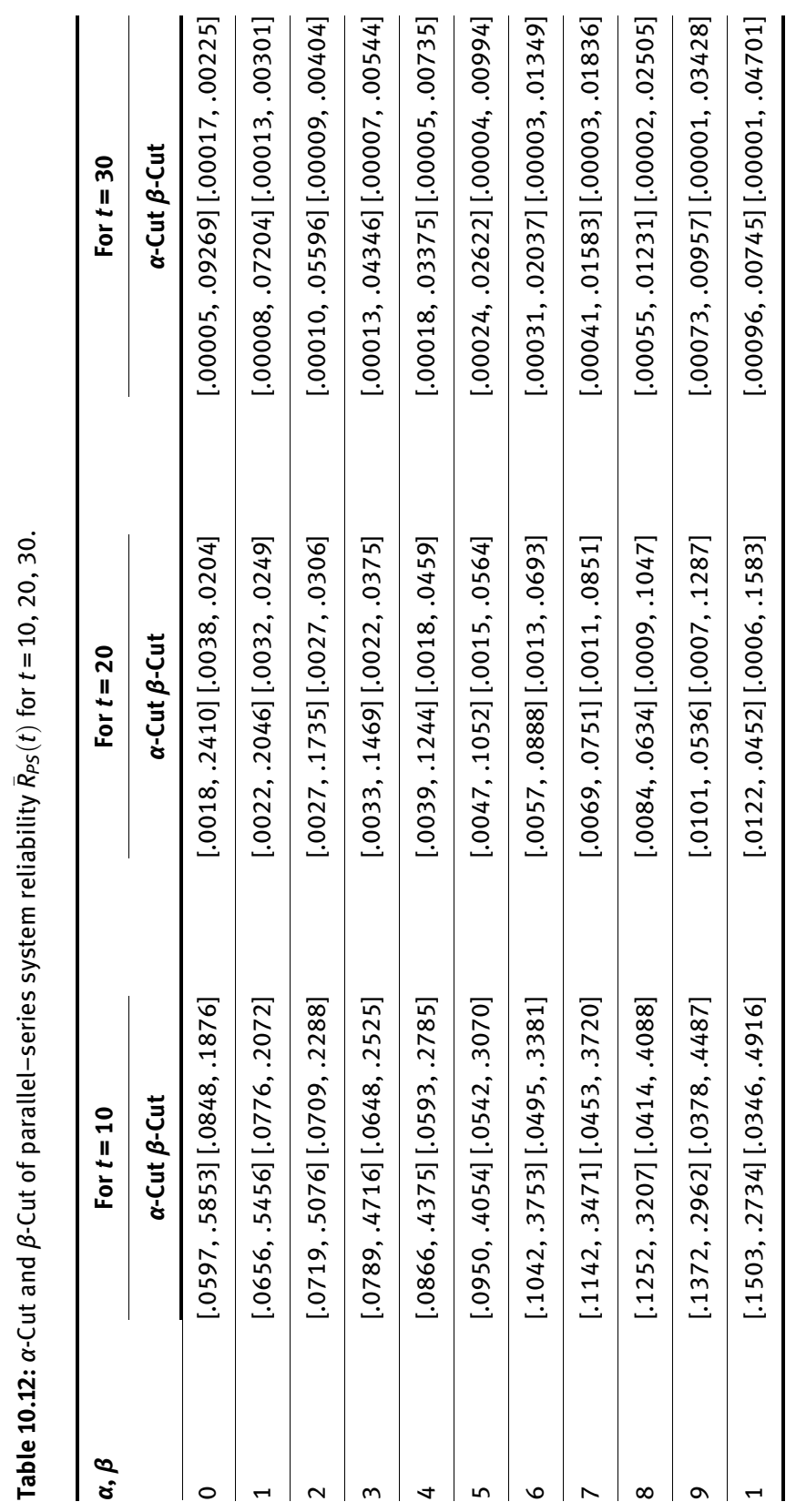




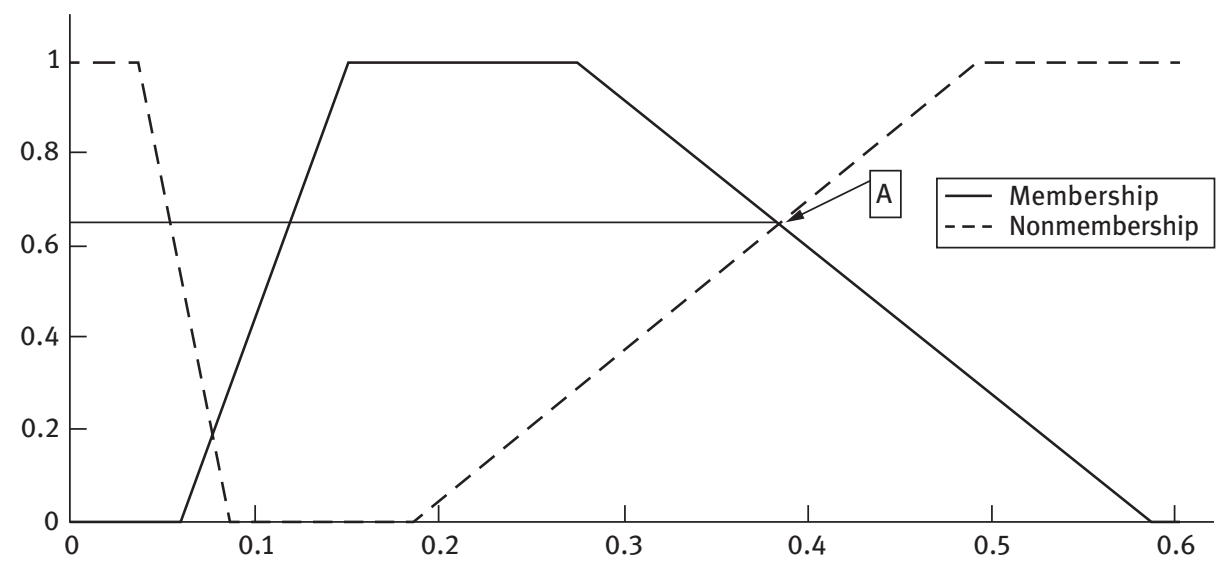

Figure 10.11: For $t=10$, membership function and nonmembership function of reliability of communication system.

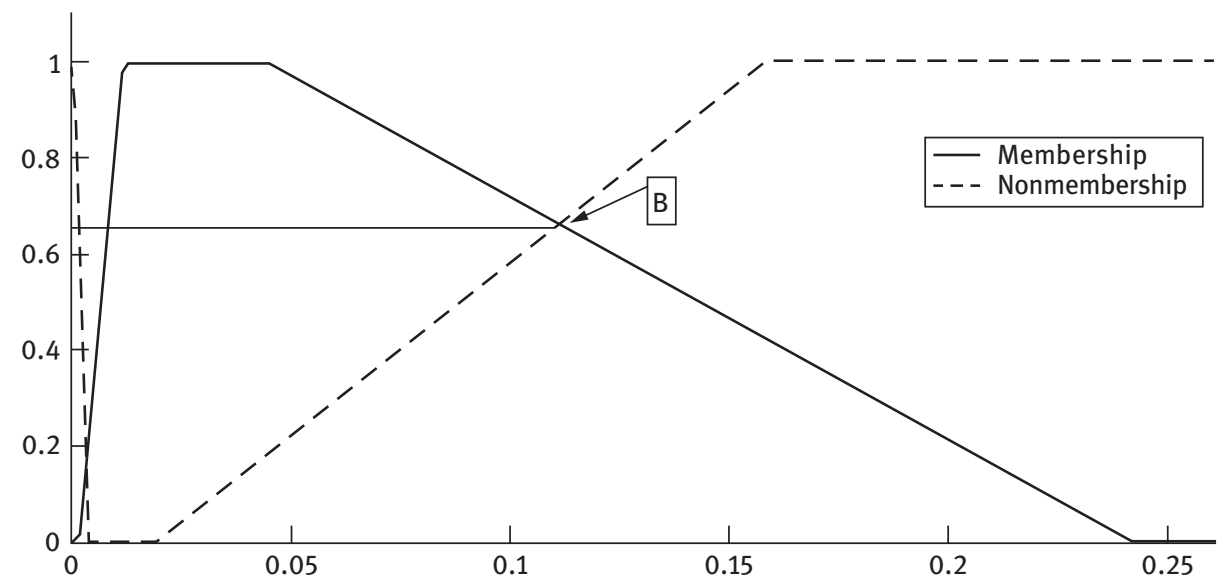

Figure 10.12: For $t=20$, membership function and nonmembership function of reliability of communication system.

Let failure rate function of the $r$ th component of $s$ th subsystem $(r=1,2, \ldots, j$ and $s=1,2, \ldots, i)$ is represented by CBFS $\bar{\lambda}_{r s}$.

Reliability of the $r$ th component of sth branch is as follows:

$$
\bar{R}_{r s}(t)=\exp \left(-\bar{\lambda}_{r s} \cdot t\right)
$$

It is well known that the fuzzy reliability of system $\bar{R}_{S P}(t)$ is given below: 


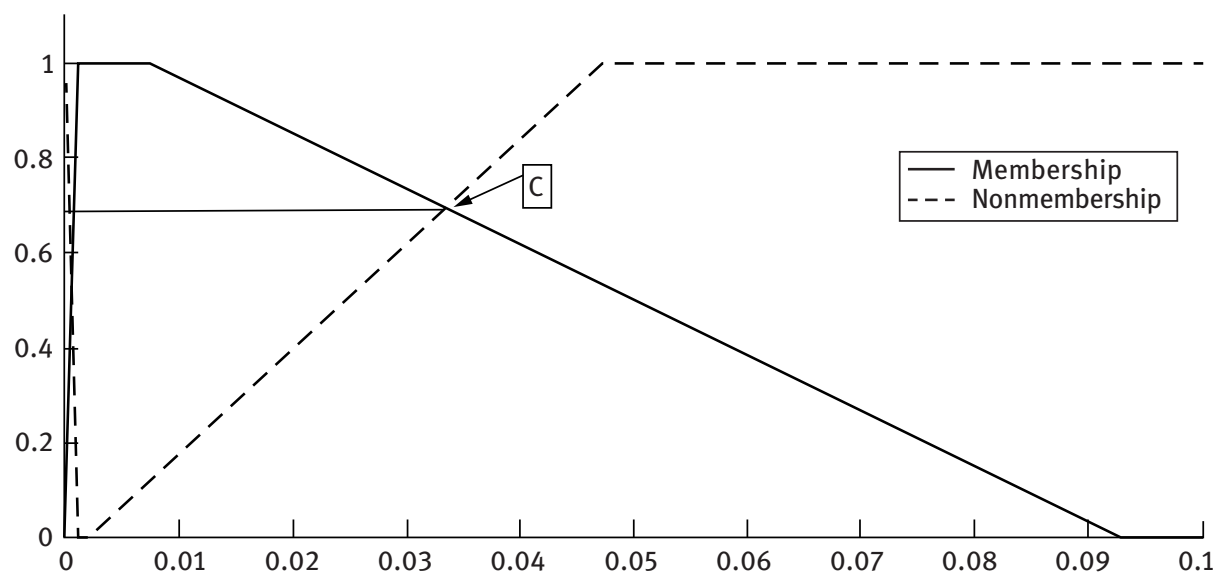

Figure 10.13: For $t=30$, membership function and nonmembership function of reliability of communication system.

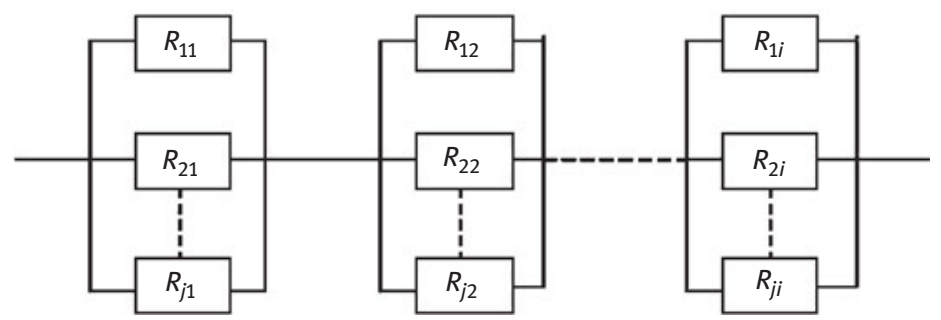

Figure 10.14: Series-parallel system.

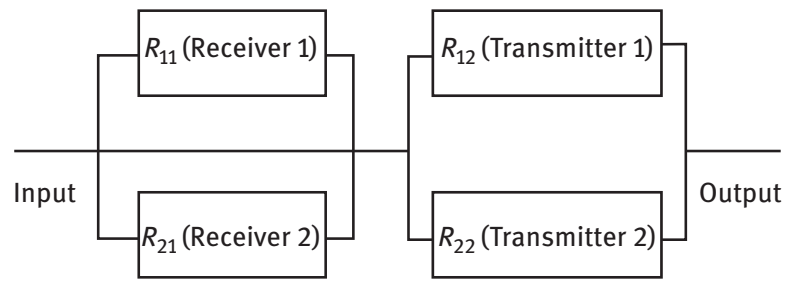

Figure 10.15: Series-parallel system. 
Table 10.13: Conflicting bifuzzy failure rates of components.

\begin{tabular}{ll}
\hline Bifuzzy failure rate & Types of bifuzzy number \\
\hline$\tilde{\lambda}_{11}=(0.06,0.09,0.12 ; 0.07,0.11,0.16)$ & Triangular conflicting bifuzzy number \\
\hline$\tilde{\lambda}_{12}=(0.07,0.12,0.17,0.22 ; 0.09,0.14,0.19,0.24)$ & Trapezoidal conflicting bifuzzy number \\
\hline$\tilde{\lambda}_{21}=(0.06,0.11,0.16 ; 0.08,0.13,0.18)$ & Triangular conflicting bifuzzy number \\
\hline$\tilde{\lambda}_{22}=(0.08,0.13,0.18,0.22 ; 0.10,0.15,0.20,0.25)$ & Trapezoidal conflicting bifuzzy number \\
\hline
\end{tabular}

Table 10.14: $\alpha$-Cut of $\tilde{\lambda}_{11}, \tilde{\lambda}_{12}, \tilde{\lambda}_{21}$, and $\tilde{\lambda}_{22}$ for membership.

\begin{tabular}{lllll}
\hline$\alpha$ & $\tilde{\lambda}_{11 \alpha}$ & $\tilde{\lambda}_{12 \alpha}$ & $\tilde{\lambda}_{21 \alpha}$ & $\tilde{\lambda}_{22 \alpha}$ \\
\hline 0.0 & {$[0.06,0.12]$} & {$[0.07,0.22]$} & {$[0.06,0.16]$} & {$[0.08,0.22]$} \\
\hline 0.1 & {$[0.063,0.117]$} & {$[0.075,0.215]$} & {$[0.065,0.155]$} & {$[0.085,0.216]$} \\
\hline 0.2 & {$[0.066,0.114]$} & {$[0.08,0.21]$} & {$[0.07,0.15]$} & {$[0.090,0.212]$} \\
\hline 0.3 & {$[0.069,0.111]$} & {$[0.085,0.205]$} & {$[0.075,0.145]$} & {$[0.095,0.208]$} \\
\hline 0.4 & {$[0.072,0.108]$} & {$[0.09,0.20]$} & {$[0.08,0.14]$} & {$[0.10,0.204]$} \\
\hline 0.5 & {$[0.075,0.105]$} & {$[0.095,0.195]$} & {$[0.085,0.135]$} & {$[0.105,0.20]$} \\
\hline 0.6 & {$[0.078,0.102]$} & {$[0.10,0.19]$} & {$[0.09,0.13]$} & {$[0.11,0.196]$} \\
\hline 0.7 & {$[0.081,0.099]$} & {$[0.105,0.185]$} & {$[0.095,0.125]$} & {$[0.115,0.192]$} \\
\hline 0.8 & {$[0.084,0.096]$} & {$[0.11,0.18]$} & {$[0.10,0.12]$} & {$[0.12,0.188]$} \\
\hline 0.9 & {$[0.087,0.093]$} & {$[0.115,0.175]$} & {$[0.105,0.115]$} & {$[0.125,0.184]$} \\
\hline 1.0 & {$[0.09,0.09]$} & {$[0.12,0.17]$} & {$[0.11,0.11]$} & {$[0.13,0.18]$} \\
\hline
\end{tabular}

$$
\bar{R}_{S P}=\prod_{s=1}^{i}\left(1-\prod_{r=1}^{j}\left(1-\bar{R}_{r S}\right)\right)=\prod_{s=1}^{i}\left(1-\prod_{r=1}^{j}\left(1-\exp \left(-\bar{\lambda}_{r s}\right)\right)\right)
$$

So $\bar{R}_{S P}(t)=\psi\left(\tilde{\lambda}_{r s}: r=1,2, \ldots, j\right.$ and $s=1,2, \ldots, i ; t=\prod_{s=1}^{i}\left\{1-\prod_{r=1}^{j}\left(1-\exp \left(-\bar{\lambda}_{r s}\right)\right)\right\}$
$\alpha$-Cut of $\tilde{\lambda}_{r s}$ for membership function is

$$
\tilde{\lambda}_{r s \alpha}=\left[h_{r s \alpha}^{L}, h_{r s \alpha}^{R}\right] \quad \forall \alpha \in[0,1], r=1,2, \ldots, j \text { and } s=1,2, \ldots, i .
$$

For every $\alpha$, we have $n^{2}$ intervals $\tilde{\lambda}_{11 \alpha}=\left[h_{11 \alpha}^{L}, h_{11 \alpha}^{R}\right], \tilde{\lambda}_{12 \alpha}=\left[h_{12 \alpha}^{L}, h_{12 \alpha}^{R}\right], \tilde{\lambda}_{21 \alpha}=\left[h_{21 \alpha}^{L}, h_{21 \alpha}^{R}\right]$, $\ldots, \tilde{\lambda}_{j i \alpha}=\left[h_{j i \alpha}^{L}, h_{j i \alpha}^{R}\right]$, for each $\tilde{\lambda}_{r s}, 1 \leq r \leq j$ and $1 \leq s \leq i$, respectively.

For nonmembership function, $\beta$-Cut of $\tilde{\lambda}_{r s}$ is 
Table 10.15: $\beta$-Cut of $\tilde{\lambda}_{11}, \tilde{\lambda}_{12}, \tilde{\lambda}_{21}$, and $\tilde{\lambda}_{22}$ for nonmembership.

\begin{tabular}{lllll}
\hline $\boldsymbol{\beta}$ & \multicolumn{1}{c}{$\tilde{\lambda}_{11 \beta}$} & \multicolumn{1}{c}{$\tilde{\lambda}_{12 \beta}$} & \multicolumn{1}{c}{$\tilde{\lambda}_{21 \beta}$} & $\tilde{\lambda}_{22 \beta}$ \\
\hline 0.0 & {$[0.11,0.11]$} & {$[0.14,0.19]$} & {$[0.13,0.13]$} & {$[0.15,0.20]$} \\
\hline 0.1 & {$[0.106,0.115]$} & {$[0.135,0.195]$} & {$[0.125,0.135]$} & {$[0.145,0.205]$} \\
\hline 0.2 & {$[0.102,0.120]$} & {$[0.13,0.20]$} & {$[0.12,0.14]$} & {$[0.14,0.21]$} \\
\hline 0.3 & {$[0.098,0.125]$} & {$[0.125,0.205]$} & {$[0.115,0.145]$} & {$[0.135,0.215]$} \\
\hline 0.4 & {$[0.094,0.130]$} & {$[0.12,0.21]$} & {$[0.11,0.15]$} & {$[0.13,0.22]$} \\
\hline 0.5 & {$[0.090,0.135]$} & {$[0.115,0.215]$} & {$[0.105,0.155]$} & {$[0.125,0.225]$} \\
\hline 0.6 & {$[0.086,0.140]$} & {$[0.11,0.22]$} & {$[0.10,0.16]$} & {$[0.12,0.23]$} \\
\hline 0.7 & {$[0.082,0.145]$} & {$[0.105,0.225]$} & {$[0.095,0.165]$} & {$[0.115,0.235]$} \\
\hline 0.8 & {$[0.078,0.150]$} & {$[0.10,0.23]$} & {$[0.09,0.17]$} & {$[0.11,0.24]$} \\
\hline 0.9 & {$[0.074,0.155]$} & {$[0.095,0.235]$} & {$[0.085,0.175]$} & {$[0.105,0.245]$} \\
\hline 1.0 & {$[0.07,0.16]$} & {$[0.09,0.24]$} & {$[0.08,0.18]$} & {$[0.10,0.25]$} \\
\hline
\end{tabular}

$$
\tilde{\lambda}_{r s \beta}=\left[h_{r s \beta}^{L}, h_{r s \beta}^{R}\right] \quad \forall \beta \in[0,1], r=1,2, \ldots, j \text { and } s=1,2, \ldots, i
$$

Similarly, for each $\beta$, we have $n^{2}$ intervals $\tilde{\lambda}_{11 \beta}=\left[h_{11 \beta}^{L}, h_{11 \beta}^{R}\right], \tilde{\lambda}_{12 \beta}=\left[h_{12 \beta}^{L}, h_{12 \beta}^{R}\right], \tilde{\lambda}_{21 \beta}=$ $\left[h_{21 \beta}^{L}, h_{21 \beta}^{R}\right], \ldots, \tilde{\lambda}_{j i \beta}=\left[h_{j i \beta}^{L}, h_{j i \beta}^{R}\right]$, for any $\tilde{\lambda}_{r s}, 1 \leq r \leq j$ and $1 \leq s \leq i$, respectively.

$\alpha$-Cut of $\tilde{R}_{S P}(t)$ for membership function is

$$
\tilde{R}_{S P \alpha}(t)=\left[\psi_{S P \alpha}^{L}(t), \psi_{S P \alpha}^{R}(t)\right]
$$

where

$$
\begin{aligned}
& \psi_{S P \alpha}^{L}(t)=\min \\
& \left\{\prod_{s=1}^{i}\left(1-\prod_{r=1}^{j}\left(1-\exp \left(-\tilde{\lambda}_{r s}\right)\right)\right)\right\}, \quad \psi_{S P \alpha}^{L}(t)=\min \left\{\prod_{s=1}^{i}\left(1-\prod_{r=1}^{j}\left(1-\exp \left(-\tilde{\lambda}_{r s}\right)\right)\right)\right\} \\
& \text { s.t. } h_{11 \alpha}^{L} \leq x_{11} \leq h_{11 \alpha}^{R}, h_{12 \alpha}^{L} \leq x_{12} \leq h_{12 \alpha}^{R}, h_{21 \alpha}^{L} \leq x_{21} \leq h_{21 \alpha}^{R}, \ldots, h_{i j \alpha}^{L} \leq x_{i j} \leq h_{i j \alpha}^{R}
\end{aligned}
$$

$\beta$-Cut of $\tilde{R}_{S P}(t)$ for nonmembership function is

$$
\tilde{R}_{S P \beta}(t)=\left[\psi_{S P \beta}^{L}(t), \psi_{S P \beta}^{R}(t)\right]
$$

where

$$
\psi_{S P \beta}^{L}(t)=\min
$$




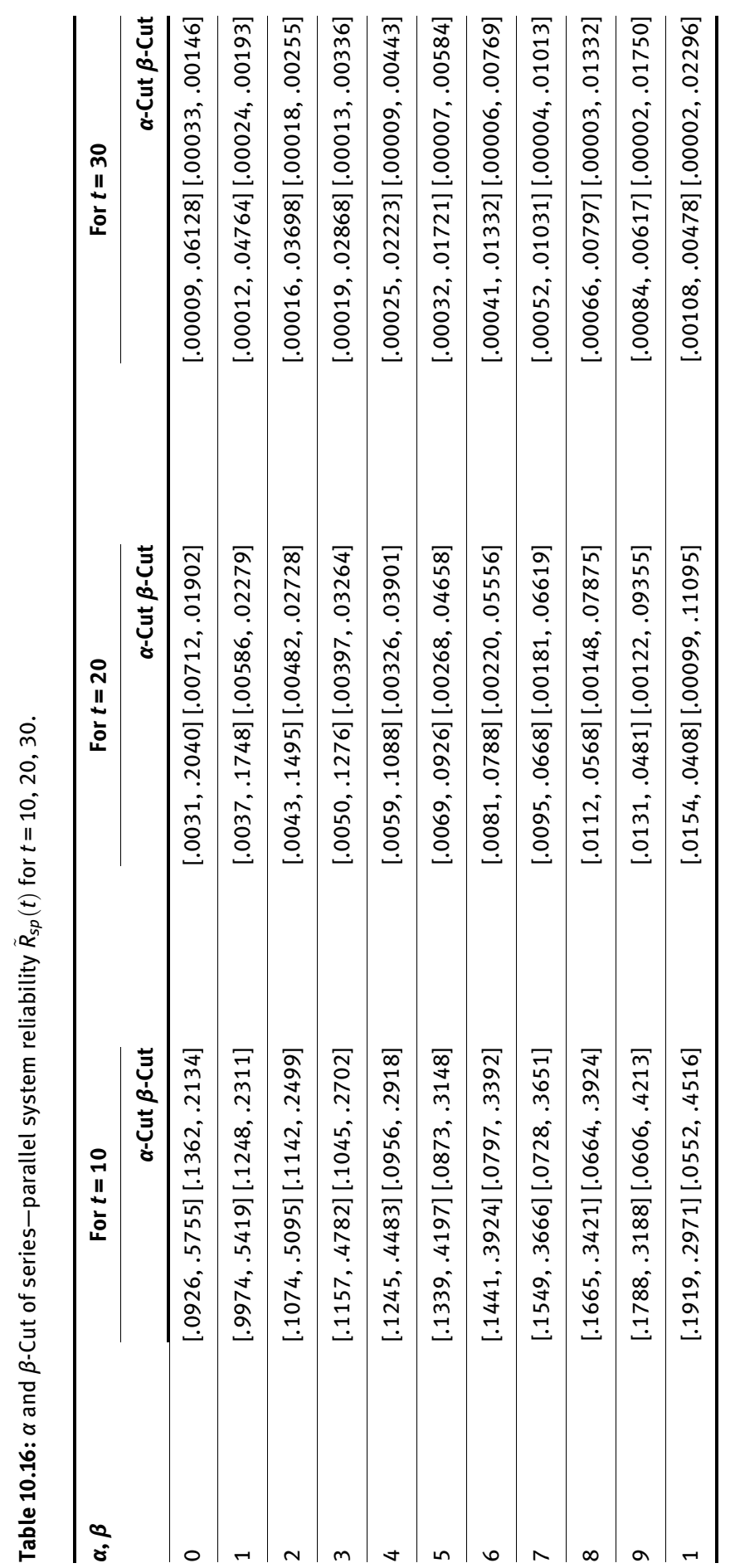




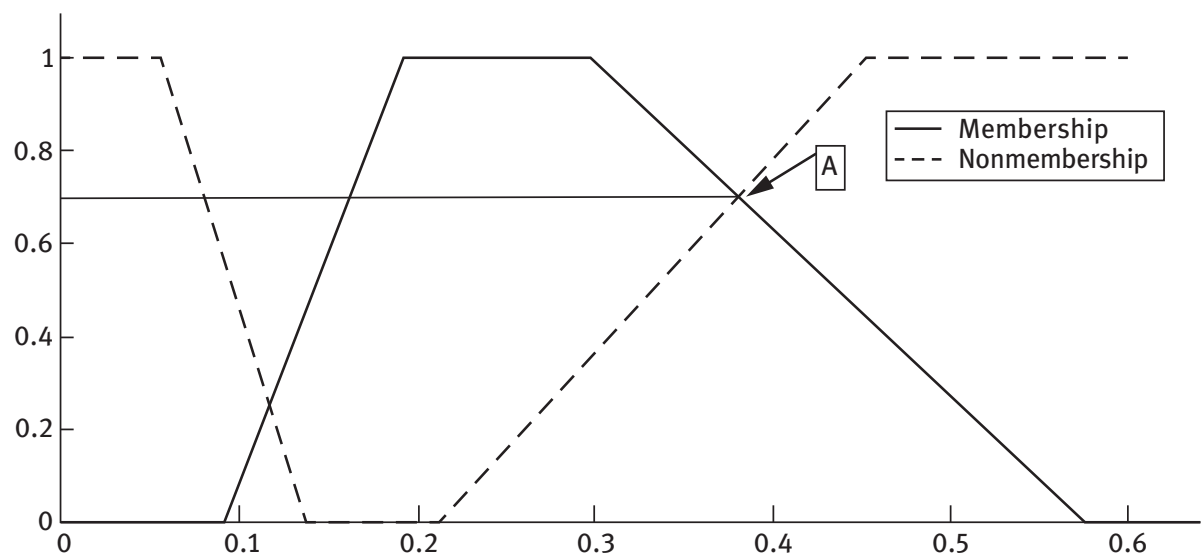

Figure 10.16: For $t=10$, membership function and nonmembership function of reliability of communication system.

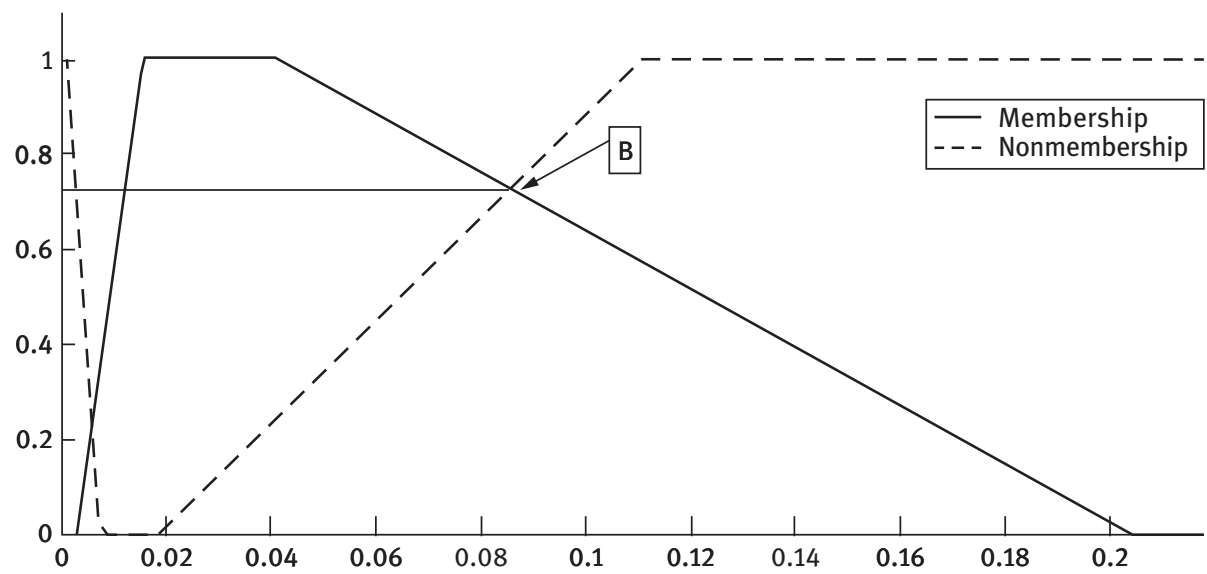

Figure 10.17: For $t=20$, membership function and nonmembership function of reliability of communication system.

$$
\begin{aligned}
& \left\{\prod_{s=1}^{i}\left(1-\prod_{r=1}^{j}\left(1-\exp \left(-\tilde{\lambda}_{r s}\right)\right)\right)\right\}, \quad \psi_{S P \beta}^{L}(t)=\min \left\{\prod_{s=1}^{i}\left(1-\prod_{r=1}^{j}\left(1-\exp \left(-\tilde{\lambda}_{r s}\right)\right)\right)\right\} \\
& \text { s.t. } h_{11 \beta}^{L} \leq x_{11} \leq h_{11 \beta}^{R}, h_{11 \beta}^{L} \leq x_{11} \leq h_{11 \beta}^{R}, h_{11 \beta}^{L} \leq x_{11} \leq h_{11 \beta}^{R}, \ldots, h_{11 \beta}^{L} \leq x_{11} \leq h_{11 \beta}^{R}
\end{aligned}
$$

After solving (10.21) and (10.22), we get out $\alpha$-Cut and $\beta$-Cut of fuzzy reliability of series-parallel system $\tilde{R}_{S P}(t)$. 


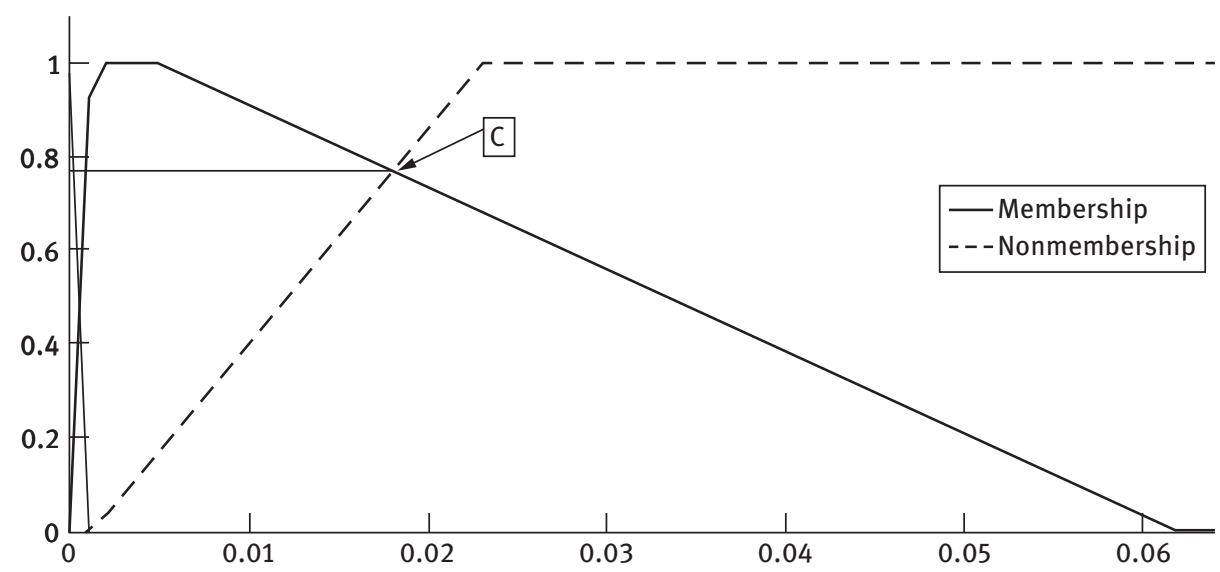

Figure 10.18: For $t=30$, membership function and nonmembership function of reliability of communication system.

\subsubsection{Numerical problem}

Suppose there is a communication system which receives the input signal and transmits the output signal. For this there are two receivers and two transmitters in the system connected as shown in Figure 10.15. For a successful communication at least one receiver and one transmitter must work properly.

The failure rate of receivers and transmitters follow different types of CBFNs as shown in Table 10.13.

The $\alpha$-Cut of $\tilde{\lambda}_{11}, \tilde{\lambda}_{12}, \tilde{\lambda}_{21}, \tilde{\lambda}_{22}$ are calculated and tabulated in Table 10.14.

The $\beta$-Cuts of $\tilde{\lambda}_{11}, \tilde{\lambda}_{12}, \tilde{\lambda}_{21}, \tilde{\lambda}_{22}$ are calculated and tabulated in Table 10.15. Using formulae (10.21) and (10.22), we get

$$
\begin{aligned}
\psi_{S P \alpha}^{L}(t)= & \left\{1-\left(1-e^{-(0.12-0.03 \alpha) t}\right) \times\left(1-e^{-(0.16-0.05 \alpha) t}\right)\right\} \times\left\{1-\left(1-e^{-(0.22-0.05 \alpha) t}\right)\right. \\
& \left.\times\left(1-e^{-(0.22-0.04 \alpha) t}\right)\right\} \\
\psi_{S P \alpha}^{R}(t)= & \left\{1-\left(1-e^{-(0.06+0.03 \alpha) t}\right) \times\left(1-e^{-(0.06+0.05 \alpha) t}\right)\right\} \times\left\{1-\left(1-e^{-(0.07+0.05 \alpha) t}\right)\right. \\
& \left.\times\left(1-e^{-(0.08+0.05 \alpha) t}\right)\right\} \\
\psi_{S P \beta}^{L}(t)= & \left\{1-\left(1-e^{-(0.11+0.05 \beta) t}\right) \times\left(1-e^{-(0.13+0.05 \beta) t}\right)\right\} \times\left\{1-\left(1-e^{-(0.19+0.05 \beta) t}\right)\right. \\
& \left.\times\left(1-e^{-(0.20+0.05 \beta) t}\right)\right\} \\
\psi_{S P \beta}^{R}(t)= & \left\{1-\left(1-e^{-(0.11-0.04 \beta) t}\right) \times\left(1-e^{-(0.13-0.05 \beta) t}\right)\right\} \times\left\{1-\left(1-e^{-(0.14-0.05 \beta) t}\right)\right. \\
& \left.\times\left(1-e^{-(0.15-0.05 \beta) t}\right)\right\}
\end{aligned}
$$


The system reliability of above system is calculated using (10.24). The system reliability is in trapezoidal CBFN for failure rate of components follow conflicting bifuzzy. The $\alpha$-Cut and $\beta$-Cut of fuzzy reliability of above system $\bar{R}_{S P}(t)$ are evaluated for $t=10,20,30$ and given in Table 10.16. The sum of membership and nonmembership degree is found to be greater than one (Figures 10.16-10.18) at points $A, B, C$.

\subsection{Conclusion}

In this study, we have discussed a new method for evaluating fuzzy reliability of different systems having components follow different types of conflicting bifuzzy failure rates. We also fabricate the membership degree and nonmembership degree of fuzzy reliability Kumar and Yadav [9]. Further, using introduced method, membership degree and nonmembership degree of fuzzy reliability of different systems like series system, parallel system, parallel-series system, and series-parallel system. The components of above systems follow different types of conflicting bifuzzy failure rates. We have seen that, the fuzzy reliabilities of above systems are in the form of trapezoidal conflicting bifuzzy numbers. The $\alpha$-Cut and $\beta$-Cut of fuzzy reliability are also evaluated at different times $(t)$.

\section{References}

[1] Zadeh L.A. (1965) Fuzzy sets, Informatics and Control, 8(3), 338-353.

[2] Zamali T., Mohd Lazim A., \& Abu Osman M.T. (2008) An introduction to conflicting bifuzzy sets theory, International Journal of Mathematics and Statistics, 86-101.

[3] Imran C.T., Syibrah M.N., \& Mohd Lazim A. (2008) A new condition for conflicting bifuzzy sets based on intuitionistic evaluation, World Academy of Science, Engineering and Technology I: Mathematical and Computational Sciences, 2(4), 161-165.

[4] Cai K.Y., Wen C.Y., \& Zhang M.L. (1991) Fuzzy variables as a basic for a theory of fuzzy reliability in the possibility context, Fuzzy Set and Systems, 42, 145-172.

[5] Cai K.Y., Wen C.Y., \& Zhang M.L. (1993) Fuzzy states as for a theory of fuzzy reliability, Microelectron Reliability, 33, 2253-2263.

[6] Chen C.H. \& Mon L. (1993) Fuzzy system reliability analysis by interval of confidence, Fuzzy Set and Systems, 56, 29-35.

[7] Onisawa T. \& Kacprzyk J. (1995) Reliability and safety analysis under fuzziness, PhysicaVerlag Heidelberg.

[8] Cai K.Y. (1996) System failure engineering and fuzzy methodology. An introductory overview, Fuzzy Set and Systems, 83, 113-133.

[9] Kumar M. \& Yadav S.P. (2012) A novel approach for analyzing fuzzy system reliability using different types of intuitionistic fuzzy failure rates of components, ISA Transactions, 51, 288-297.

[10] Aliev M. \& Kara Z. (2004) Fuzzy system reliability analysis using time dependent fuzzy set, Control and Cybernetics, 33, 653-662. 
[11] Mon D.L. \& Cheng C.H. (1994) Fuzzy system reliability analysis for components with different membership functions, Fuzzy Sets and Systems, 64, 145-157.

[12] Kumar M., Yadav S.P., \& Kumar S. (2011) A new approach for analyzing the fuzzy system reliability using intuitionistic fuzzy number, International Journal of Industrial and Systems Engineering, 8(2), 135-156. 